\title{
Nueva asociación de mamíferos del Paleógeno en el este de la Patagonia (provincia de Chubut, Argentina): implicancias biocronológicas y paleobiogeográficas
}

\author{
*María Teresa Dozo ${ }^{1,2}$, Martín Ciancio ${ }^{1,3,4}$, Pablo Bouza ${ }^{1,2}$, Gastón Martínez ${ }^{1,2}$ \\ ${ }^{I}$ CONICET (Consejo Nacional de Investigaciones Científicas y Técnicas), Avda. Rivadavia 1917 (C1033AAJ), Ciudad Autónoma de \\ Buenos Aires, Argentina. \\ ${ }^{2}$ Centro Nacional Patagónico, 9120 Puerto Madryn, Argentina. \\ dozo@cenpat.edu.ar,bouza@cenpat.edu.ar,gmartinez@cenpat.edu.ar \\ ${ }^{3}$ División Paleontología de Vertebrados, Museo de La Plata, Paseo del Bosque s/n B1900FWA La Plata, Argentina. \\ ${ }^{4}$ Cátedra de Anatomía Comparada, Facultad de Ciencias Naturales y Museo, Universidad Nacional de La Plata, Avenida 122 y 60 , \\ La Plata, Argentina. \\ mciancio@fcnym.unlp.edu.ar \\ *Autor de correspondencia: dozo@cenpat.edu.ar
}

RESUMEN. Los fósiles de mamíferos continentales hallados en la localidad Barrancas Blancas, ubicada en la Estancia 'La Argentina', cerca de Camarones, en el sureste de la provincia del Chubut, Argentina, constituyen una nueva asociación faunística del Paleógeno de Patagonia. El afloramiento en dicha localidad está integrado, desde la base al techo, por sedimentitas continentales y marinas asignadas a la Formación Sarmiento y a la Formación Chenque, respectivamente. En la sección inferior de la Formación Sarmiento se encontraron mamíferos fósiles, principalmente Xenarthra, Notoungulata y Litopterna, que conforman la nueva asociación. Se han podido reconocer los siguientes taxones: Xenarthra, Cingulata, Dasypodidae, Euphractinae, Eutatini: Meteutatus percarinatus; Euphractini: Archaeutatus sp.; Parutaetus; Prozaedyus; Peltephilidae: Peltephilus undulatus; Glyptodontidae, Glyptatelinae indet.; Cingulata incertae sedis: Machlydotherium ater; Phyllophaga: Pseudoglyptodon; Notoungulata, 'Notohippidae'; 'Rhynchippinae': Rhynchippus pumilus; 'Rhynchippinae' indet.; Interatheriidae: Eopachyrucos pliciferus, Proargyrohyrax curanderensis; 'Archaeohyracidae': Pseudhyrax eutrachytheroides?; Leontiniidae: Leontinia gaudryi?; Isotemnidae indet.; Litopterna, Proterotheriidae indet. Los nuevos hallazgos paleontológicos confirmarían en principio una edad Mamífero Deseadense para estos niveles por la presencia de los géneros Leontinia y Rhynchippus. Sin embargo, la diversidad de xenartros y de varios grupos de tipoterios (Interatheriidae y 'Archaeohyracidae') presentes en Barrancas Blancas indicarían una fauna de EM Tinguiriquense o incluso podría estar representando una fauna equivalente a los niveles de La Cantera (Gran Barranca), cuyas dataciones de ca. $30 \mathrm{Ma}$ estarían indicando un lapso pos-Tinguiririquense a pre-Deseadense. Desde el punto de vista paleobiogeográfico, Barrancas Blancas correspondería a la localidad patagónica más oriental con fauna paleógena de las edades mencionadas anteriormente.

Palabras clave: Tinguiririquense, Deseadense, Mamiferos, Paleógeno, Chubut, Patagonia, Argentina. 


\begin{abstract}
New association of Paleogene Mammals in Eastern of Patagonia (Chubut Province, Argentina): biochronological and paleobiogeographical implications. The continental fossil mammals found in Barrancas Blancas, (near Camarones, Southeast of Chubut Province, Argentina) constitute a new faunal association from the Paleogene of Patagonia. From the base to the top, the outcrop in this locality is composed of continental and marine sedimentites, assigned to Sarmiento Formation and Chenque Formation, respectively. The fossil mammals that represent the new association (mainly Xenarthra, Notoungulata and Litopterna) come from the lower section of the Sarmiento Formation. The following taxa have been recognized: Xenarthra, Cingulata, Dasypodidae, Euphractinae, Eutatini: Meteutatus percarinatus; Euphractini: Archaeutatus sp.; Parutaetus; Prozaedyus; Peltephilidae: Peltephilus undulatus; Glyptodontidae, Glyptatelinae indet.; Cingulata incertae sedis: Machlydotherium ater; Phyllophaga: Pseudoglyptodon; Notoungulata, 'Notohippidae', 'Rhynchippinae': Rhynchippus pumilus; 'Rhynchippinae' indet.; Interatheriidae: Eopachyrucos pliciferus, Proargyrohyrax curanderensis; 'Archaeohyracidae': Pseudhyrax eutrachytheroides?; Leontiniidae: Leontinia gaudryi?; Isotemnidae indet.; Litopterna, Proterotheriidae indet. Although the presence of Leontinia and Rhynchippus might support a Deseadan age for these levels, the high diversity of xenarthrans and many groups of tipotheres (Interatheriidae and Archaeohyracidae) could indicate a Tinguirirican mammal Age. Moreover, this association could represent an equivalent fauna to that registered in levels from La Cantera (Gran Barranca), with a radiometric estimated age of $c a .30 \mathrm{Ma}$, which would indicate a post-Tinguirirican to pre-Deseadan age. From a paleobiogeographic point of view Barrancas Blancas would correspond to the most eastern Patagonian locality with Paleogene Fauna of the ages mentioned above.
\end{abstract}

Keywords: Tinguirirican, Deseadan, Mammals, Paleogene, Chubut, Patagonia, Argentine.

\section{Introducción}

Las localidades del Paleógeno del centro y centro sur de la provincia de Chubut fueron estudiadas intensamente en los últimos 25 años por varios grupos de investigación (e.g., Cifelli, 1985; Marshall et al., 1986; Bown y Fleagle, 1993; Kay et al., 1999), destacándose recientemente los exhaustivos trabajos en la localidad Gran Barranca (Madden et al., 2010). Con el propósito de completar y acrecentar el conocimiento de los mamíferos paleógenos de Patagonia, sobre todo del Oligoceno, se han llevado a cabo trabajos en localidades del sureste de Chubut donde hay afloramientos asignados a la Formación Sarmiento (Feruglio, 1949). Tal es el caso de Cabeza Blanca (Loomis, 1914; Feruglio, 1949; Bown et al., 1993; Reguero et al., 2007; Marani y Dozo, 2008; Dozo y Vera, 2010), en el Departamento Escalante, localidad que no había sido estudiada con continuidad, y otras nuevas como Barrancas Blancas, en las cercanías de Camarones en el Departamento Florentino Ameghino (Lema et al., 2001). En dicha localidad, al igual que en Cabeza Blanca, afloran sedimentitas continentales y marinas correspondientes, las primeras, a la Formación Sarmiento y, las segundas, al 'Patagoniano'. Los escasos restos de mamíferos fósiles recolectados en la zona por Lema et al. (2001) habían indicado prima facie una Edad Mamífero (EM) Deseadense para la fauna; sin embargo, las nuevas y más completas evidencias encontradas en la sección inferior de la Formación Sarmiento estarían señalando también una edad más antigua, posiblemente de Edad Mamífero (EM) Tinguiririquense. La localidad tipo de esta EM fue establecida en Chile central (Flynn et al., 2003) a la que se sumaron varias faunas de Patagonia Argentina, la mayoría pobremente muestreadas y en un caso, como es Cañadón Blanco, sin poder ser relocalizada (Croft et al., 2008).

Barrancas Blancas constituye una nueva localidad con fauna paleógena en la Patagonia oriental y en donde no solo podría estar representada la EM Deseadense, sino también la EM Tinguiririquense y lapso pos-Tinguiririquense a pre-Deseadense.

La presente contribución tiene por objetivos: 1 . dar a conocer una nueva asociación de mamíferos fósiles continentales del Paleógeno para el sureste de la provincia de Chubut, y 2. considerar su edad y las posibles implicancias bioestratigráficas, biocronológicas y paleobiogeográficas que puede aportar dicha asociación faunística al conocimiento de los mamíferos del Paleógeno de Patagonia.

\section{Antecedentes}

\subsection{Edad Mamífero Tinguiririquense}

La EM Tinguiririquense fue establecida formalmente por Flynn et al. (2003) sobre la base de 25 taxa provenientes de un conjunto faunístico de los Andes chilenos y otras faunas correlativas de la Patagonia Argentina, correspondientes a Cañadón Blanco (Roth, 1901, 1903), los niveles del 'Astraponotense más 
superior' (APS) de Gran Barranca (Ameghino, 1901, 1902), en la provincia de Chubut, y Rocas Bayas en Río Negro. Sin embargo, Bond et al. (1996, 1997a, b) consideraron que no serían estrictamente sincrónicas, ya que se advierten diferencias composicionales entre estas faunas locales. Reguero (1999) comentó que, aunque carece de dataciones geocronológicas, la fauna APS parece ser más antigua que la de Tinguiririca (localidad tipo de la EM Tinguiririquense), debido a una mayor presencia de taxones comunes con los de la EM Mustersense. Consideró, además, que las faunas del APS de Gran Barranca, las de Cañadón Blanco, Lomas Blancas ( $=$ La Curandera) y Rinconada de los López pertenecerían a este intervalo y solo la fauna de Rocas Bayas sería coetánea a la de Tinguiririca.

Se debe destacar que para la EM Tinguiririquense se obtuvieron dataciones que indican una edad de 31,5 Ma para los niveles portadores de la localidad tipo, aunque Flynn et al. (2003) incluyen también un espectro que va desde los 37 a $31 \mathrm{Ma}, \mathrm{y}$ ca. $33,5 \mathrm{Ma}$ para los niveles equivalentes en Gran Barranca- $\mathrm{La}$ Cancha (Ré et al., 2010). Esta EM estaría completando parcialmente el intervalo de 15-25 millones de años que precede a la EM Deseadense y representaría la primera fauna posterior a la transición Eoceno-Oligoceno (TEO). En la TEO se registra un abrupto descenso de las temperaturas a gran escala ('Oi-1 glaciation'; Zachos et al., 2001) producto de un cambio en el sistema de circulación oceánica (Prothero, 1994; Dingle y Lavelle, 1998; Salamy y Zachos, 1999). Este descenso de temperaturas habría producido un deterioro climático que afectó a las biotas mundiales, impulsando un recambio biológico masivo catalogado como 'La Grande Coupure' en Europa (Stehlin, 1909), 'Terminal Eocene Event' en América del Norte (Wolfe, 1978) o 'Patagonian Hinge' en el cono sur sudamericano (Goin et al., 2010). Croft et al. (2008) acuerdan en señalar que la EM Tinguiririquense marca las primeras etapas en la modernización de la fauna de mamíferos sudamericanos. Entre sus taxones, la fauna de Tinguiririca incluye una gran diversidad de especies de tipo 'rodentiforme' y una de las asociaciones de herbívoros más antiguas del mundo, dominadas por especies con dentición hipsodonta.

\subsection{Edad Mamífero Deseadense}

La EM Deseadense (Oligoceno tardío) representa un intervalo muy significativo desde el punto de vista de la evolución de los mamíferos de América del Sur durante el Cenozoico, ya que, como expresan varios autores (Pascual, 1984; Pascual et al., 1985; Flynn y Swisher III, 1995; Pascual et al., 1996; Bond et al., 1998), durante ese lapso se termina de definir la 'modernización' de la fauna de mamíferos sudamericanos. En este sentido los acontecimientos más destacados son el primer registro de primates (solo en Bolivia), el registro más antiguo que atestigua la primera radiación de los roedores caviomorfos en Patagonia (Vucetich et al., 2010) y la aparición de nuevos linajes dentro de la mayor parte de los grupos endémicos (e.g., notoungulados, litopternos, xenarthros y marsupiales). Un dato notable en la fauna deseadense es la variedad de mamíferos con grandes tamaños corporales (e.g., Pyrotherium, Proborhyaena, Scarritia y Leontinia).

Los principales yacimientos paleontológicos portadores de fauna deseadense se localizan en Patagonia Argentina- Chubut, Santa Cruz (Marshall et al., 1983; Carlini et al., 2009) y en Bolivia -Salla (Marshall y Sempere, 1991), aunque también se registran en otras zonas de Argentina: Mendoza (Cerdeño, 2011), Entre Ríos y Corrientes (Bond et al., 1998 y referencias citadas allí); Brasil (Vucetich y Ribeiro, 2003), Uruguay (Ubilla et al., 1994), Chile (Croft et al., 2008) y Perú (Shockey et al., 2009).

Aunque Ameghino (1889) ya había descrito restos de mamíferos de la EM Deseadense para el 'Neuquén', entre ellos precisamente Pyrotherium, además de Trachytherus, el punto de partida del conocimiento de la fauna deseadense (couches à Pyrotherium) (Ameghino, 1894a; Tournoüer, 1903) fue un conjunto de fósiles que colectara Carlos Ameghino durante su séptima expedición a Patagonia (1893-1894), en una localidad denominada La Flecha, en el NE de Santa Cruz. Luego, en 1903, el coleccionista francés A. Tournoüer también colectó en La Flecha una serie de restos de mamíferos que fueron estudiados por A. Gaudry (1906), sobre la base de los cuales propuso el 'Etage du Deseado', equivalente en parte al 'couches à Pyrotherium' de F. Ameghino (1894a, 1897, 1906), reconocido posteriormente como Edad Deseadense (Feruglio, 1949; Wood y Patterson, 1959), y por Pascual et al. (1965) y, hasta hoy, como EM Deseadense (Pascual et al., 2002).

Aunque la localidad tipo es La Flecha, la fauna mejor conocida de la EM Deseadense proviene de la localidad Cabeza Blanca descubierta en 1896 por Carlos Ameghino (Windhausen, 1931). Dicha 
localidad fue estudiada entre otros por Loomis (1914), Simpson (1941), Bordas (1943), Feruglio (1949), Bown et al. (1993), Reguero y Escribano (1996), Reguero et al. (2007), Marani y Dozo (2008), Dozo y Vera (2010). Esta localidad ha sido usada muchas veces, junto con La Flecha, como referencia primaria en el estudio de otras faunas deseadenses del resto de América del Sur y ha servido como base operacional para definir y caracterizar a esta EM (Marshall et al., 1986).

Para precisar su antigüedad, se han realizado diversas dataciones radioisotópicas sobre las secuencias estratigráficas portadoras de estas faunas (Marshall y Pascual, 1977, 1978; Marshall et al., 1986, Flynn y Swisher III, 1995). Los datos radiométricos, tomados en Bolivia, ubican a la Edad Deseadense entre 29,4 y 25,8 Ma (Kay et al., 1998), considerándose 28,1-27,4 Ma para las secuencias equivalentes en Gran Barranca, Patagonia (Ré et al., 2010). Sin embargo, las localidades patagónicas asignadas al Deseadense no serían sincrónicas y podrían estar representando diferentes lapsos dentro del Oligoceno tardío (ver Ciancio, 2010). De esta manera, los nuevos datos geocronológicos están restringiendo la EM Deseadense casi enteramente al Oligoceno tardío. Sin embargo, la mayor parte de las faunas asignadas a este lapso no han sido datadas todavía, especialmente la localidad tipo de La Flecha, lo que permitiría precisar la real antigüedad de la EM Deseadense, por lo que aún quedan incertidumbres en cuanto a la verdadera extensión cronológica de esta EM. Por otra parte, la correlación entre localidades deseadenses patagónicas y entre las de Bolivia y Patagonia dista bastante de haberse dilucidado. Sin embargo, considerando la información que ofrecen los Cingulata y los Notoungulata, podemos suponer que las faunas de los afloramientos del Oligoceno de Bolivia podrían estar más relacionadas con aquellas del Oligoceno de la Mesopotamia y Uruguay (Formación Fray Bentos) que con aquellas de Patagonia de edades equivalentes (Carlini y Scillato-Yané, 1999; Bond et al., 1998; Ciancio, 2010).

\section{Materiales y Métodos}

Simultáneamente a los trabajos paleontológicos, se realizó el trabajo geológico de apoyo, a través del levantamiento de un perfil estratigráfico de detalle, correspondiente a la localidad Barrancas Blancas para analizar las litofacies y realizar el muestreo de rocas para estudios sedimentológicos, con la finalidad de aportar datos en las reconstrucciones paleoambientales.

La determinación de los ejemplares, depositados en el Museo Paleontológico Egidio Feruglio (MPEF-PV), se efectuó sobre la base de la comparación anatómica con materiales pertenecientes a las colecciones de distintos museos argentinos (MACN, MLP). Para la correlación geocronológica de las EM se ha seguido a Flynn y Swisher III (1995) y Madden et al. (2010).

Abreviaturas. C: canino superior; Der.: derecho; Ix: incisivos superiores; Izq.: izquierdo; LL: diámetro labio-lingual; $\mathbf{M}$ : molar superior; $\mathbf{m}$ : molar inferior; MD: diámetro mesio-distal; P: premolar superior; p: premolar inferior.

Acrónimos. MACN: Museo Argentino de Ciencias Naturales Bernardino Rivadavia (Ciudad Autónoma de Buenos Aires); MLP: Museo de La Plata (La Plata, Buenos Aires); MPEF-PV: Museo Paleontológico Egidio Feruglio, colección Paleontología Vertebrados (Trelew, Chubut).

\section{Contexto geográfico y estratigráfico}

La localidad Barrancas Blancas, ubicada en el límite occidental de la Hoja Geológica de Camarones (Lema et al., 2001), se localiza en la Estancia La Argentina, a unos $30 \mathrm{~km}$ de la localidad costera de Camarones, en el Departamento Florentino Ameghino en el sureste de la provincia de Chubut (Fig. 1). El afloramiento en dicha localidad está integrado, desde la base al techo, por sedimentitas continentales y marinas correspondientes a la Formación Sarmiento, las primeras, y a la Formación Chenque (Sciutto et al., 2000; Patagoniano sensu Feruglio, 1950), las segundas. Esta última se ha denominado también Formación Camarones (Camacho, 1979). Coronan estas secuencias los Rodados Patagónicos denominados como Formación Montemayor (Yllañez, 1979).

El perfil sedimentario relevado tiene un total aproximado de $90 \mathrm{~m}$ y en él se pueden reconocer siete niveles principales (Fig. 2). Desde la base, los primeros cuatro corresponden a la Formación Sarmiento, los dos siguientes a la Formación Chenque y el último a la Formación Montemayor (Rodados Patagónicos). La base está cubierta o parcialmente cubierta, y se observan areniscas macizas de color blanco amarillento de consistencia dura con frecuentes nódulos blancos extremadamente duros. 


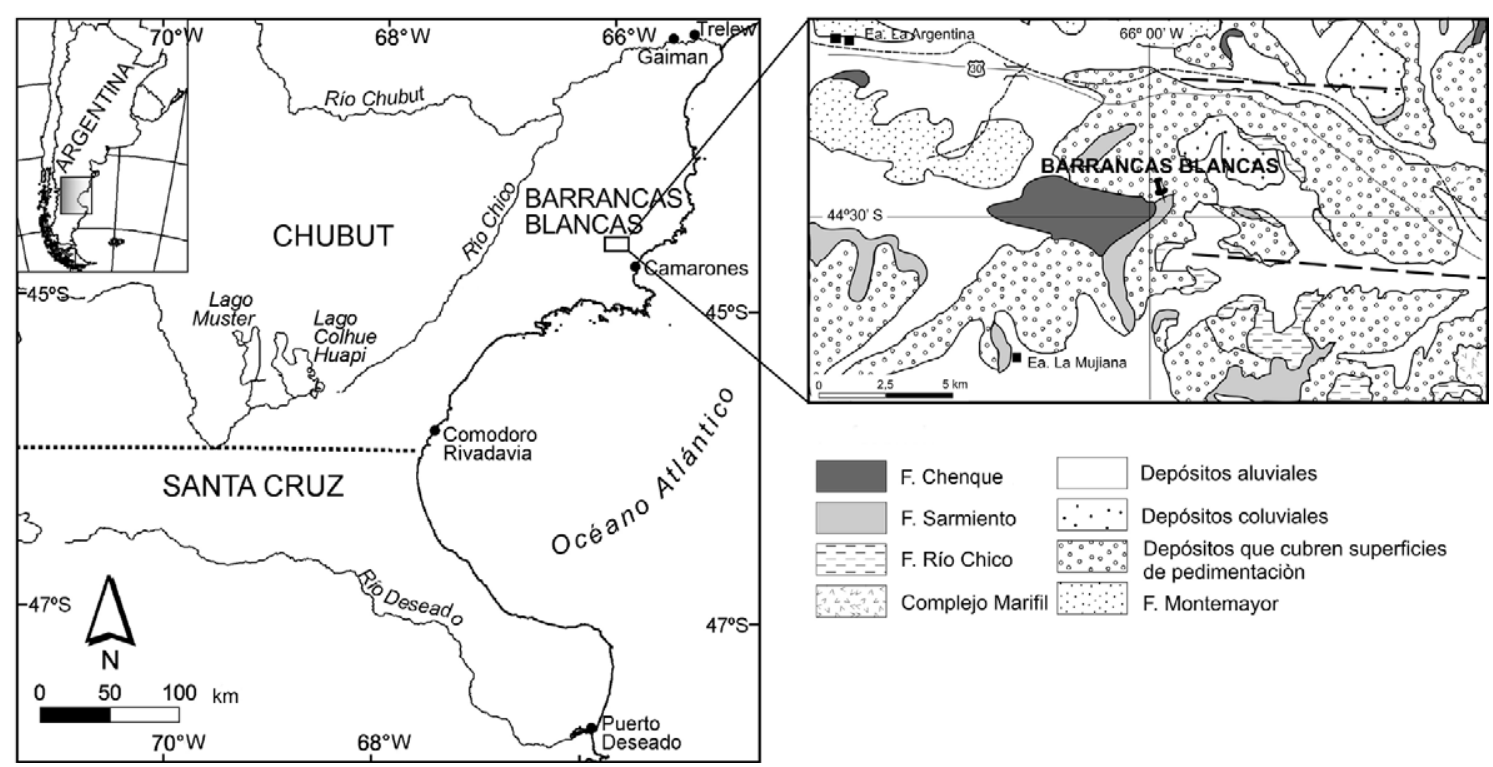

FIG. 1. Ubicación geográfica y mapa geológico de la localidad Barrancas Blancas, provincia de Chubut, Argentina, donde fueron encontrados los especímenes descritos.

El nivel 1 se caracteriza por la presencia de tobas finas macizas y de segundo ciclo (retransportadas) con restos de fósiles de mamíferos continentales. Se asocian intercalaciones de limolitas y areniscas finas y medias con laminación paralela, ocasionales lentes conglomerádicos intraformacionales y niveles de minerales opacos. Mediante contacto erosivo, se deposita por encima el nivel 2, caracterizado por pelitas de color rosa claro con laminación paralela y limolitas de color castaño claro con tonalidades verdes. Hacia la parte superior, la secuencia pasa a arcillitas macizas de color castaño rojizo oscuro. El contacto con el nivel 3 es neto y resalta la presencia de yeso. Este nivel se caracteriza por las areniscas grises con estratificación paralela y entrecruzada con niveles de minerales opacos. Se observan, además, intercalaciones de lentes de conglomerados intraformacionales y de bancos tabulares de arcillitas castañas rojizas de hasta $7 \mathrm{~cm}$ de espesor. Hacia el tope, se destacan en este nivel paleosuelos superpuestos con abundantes huecos de tipo canales (paleosuelo con desarrollo de bioturbaciones o pedotúbulos) y muy escasos nidos de escarabajos estercoleros (icnogénero Coprinisphaera, Bellosi et al., 2010). El nivel 4, sin estructura en la base, se caracteriza por la presencia de areniscas de color rojizo y lentes conglomerádicos y arenosos. Se observan, además, abundantes muñecos de tosca en la superficie. El nivel 5 presenta areniscas bioclásticas de color gris amarillento, moderadamente consolidadas, sin estructura. Se observan frecuentes rellenos de bioturbaciones tubulares de distribución vertical. El tope se encuentra cubierto con material coluvial. El nivel 6 presenta intercalaciones de bancos duros de coquinas de color castaño amarillento compuestos fundamentalmente por turritelas y areniscas grises bioclásticas y arcillitas. En el tope de los bancos duros se observa el desarrollo de megaóndulas de amplitud máxima de hasta $50 \mathrm{~cm}$. En la base de los bancos duros, se observa un contacto erosivo con abundantes moldes de carga y bioturbaciones y estructuras de corte y relleno. Las areniscas son macizas, medianamente consolidadas y presentan escasas conchillas fragmentadas e intercalaciones de hasta $30 \mathrm{~cm}$ de espesor de arcillitas de color gris verdoso con laminación fina y manchas de óxidos de hierro. El nivel 7 corresponde a la Formación Montemayor (Rodados Patagónicos), compuesta por areniscas muy gruesas a sabulita que forman bancos endurecidos con cierta estratificación entrecruzada grosera. En sectores, se observan intercalaciones de areniscas finas a medianas friables. Hacia el tope se observan depósitos de conglomerados de gravas arenosas con recubrimientos calcíticos sobre gravas y acumulaciones de carbonatos. 


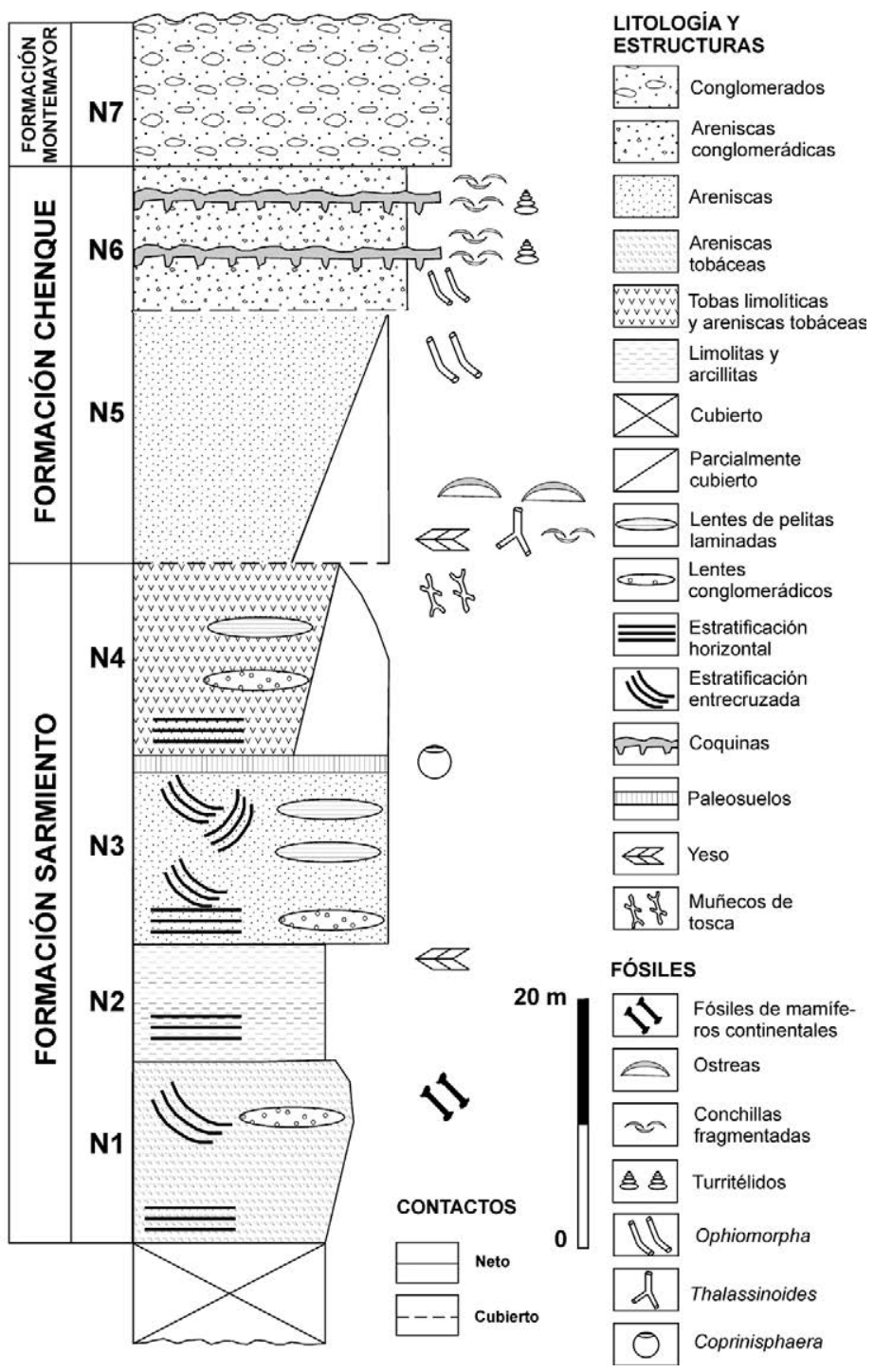

FIG. 2. Perfil estratigráfico de la localidad Barrancas Blancas, provincia de Chubut, Argentina.

El paleoambiente general de la Formación Sarmiento corresponde a una cuenca de acumulación eólica de material piroclástico al que se sobreimpuso un ambiente de llanura fluvial con cursos efímeros de notoria migración lateral. La presencia y diversidad de estructuras sedimentarias (megaóndulas, calcos de carga, corte y relleno) en la Formación Chenque evidencian un paleoambiente marino de alta energía cercano a la costa. En el sector más oriental, la secuencia marina está coronada por depósitos glaciofluviales de gravas plio-pleistocenas correspondientes a la Formación Montemayor (Rodados Patagónicos).

\section{Descripciones sistemáticas}

Clase MAMMALIA Linnaeus, 1758 Superorden XENARTHRA Cope, 1889 Orden CINGULATA Illiger, 1811 Familia DASYPODIDAE Gray, 1821 Subfamilia EUPHRACTINAE Winge, 1923 Tribu EUTATINI Bordas, 1933

Género Meteutatus Ameghino, 1902

Especie tipo: Meteutatus lagenaformis (Ameghino, 1897)

Meteutatus percarinatus Ameghino, 1902 Figura 3.1 
Material referido: MPEF-PV 2546/1-3, tres osteodermos fijos de la coraza dorsal; MPEF-PV 2546/4-6, tres osteodermos móviles.

Descripción: Los osteodermos muestran el diseño típico de Meteutatus (e.g., figura principal lageniforme, con la porción más anterior que se continúa en forma de carena medial sobre la parte posterior de la figura). Sobre el margen posterior, se observa un surco transversal divido por medio de tabiques gruesos en cavidades menores (en general cinco). Los osteodermos son más pequeños (aproximadamente un $20 \%$ ) que los de las otras especies válidas del género: M. lagenaformis Ameghino, 1902, M. concavus Ameghino, 1902 y M. lucidus Ameghino, 1902 (ver Ciancio y Carlini, 2008; Carlini et al., 2009; Carlini et al., 2010). Los osteodermos típicos del área central del escudo pélvico miden 14-17 mm de largo, 9-12 mm de ancho y 4-6 mm de grosor. Además del tamaño, el carácter más conspicuo de $M$. percarinatus es el desarrollo de la carena medial, más angosta y elevada que en las otras especies, y en algunos osteodermos fijos se observan forámenes sobre la porción anterior del surco que rodea la figura principal.

Comentarios: El material tipo de Meteutatus percarinatus se encuentra extraviado, pero la descripción de Ameghino (1902) es suficiente para poder asignar estos restos. Ameghino asigna temporalmente la especie a la 'parte superior de las capas con Notostylops' (Ameghino, 1902). Sin embargo, no se ha hallado este taxón en sedimentos, ciertamente, portadores de fauna de edades previas al Tinguiririquense, por lo que podría tratarse de un error de asignación estratigráfica (Simpson, 1948; Carlini et al., 2010). Otros ejemplares que se pueden asignar a esta especie corresponden a aquellos descritos como Meteutatus sp. (MPEF-PV 5438) en Carlini et al. (2010) para los niveles tinguiririquenses de Gran Barranca.
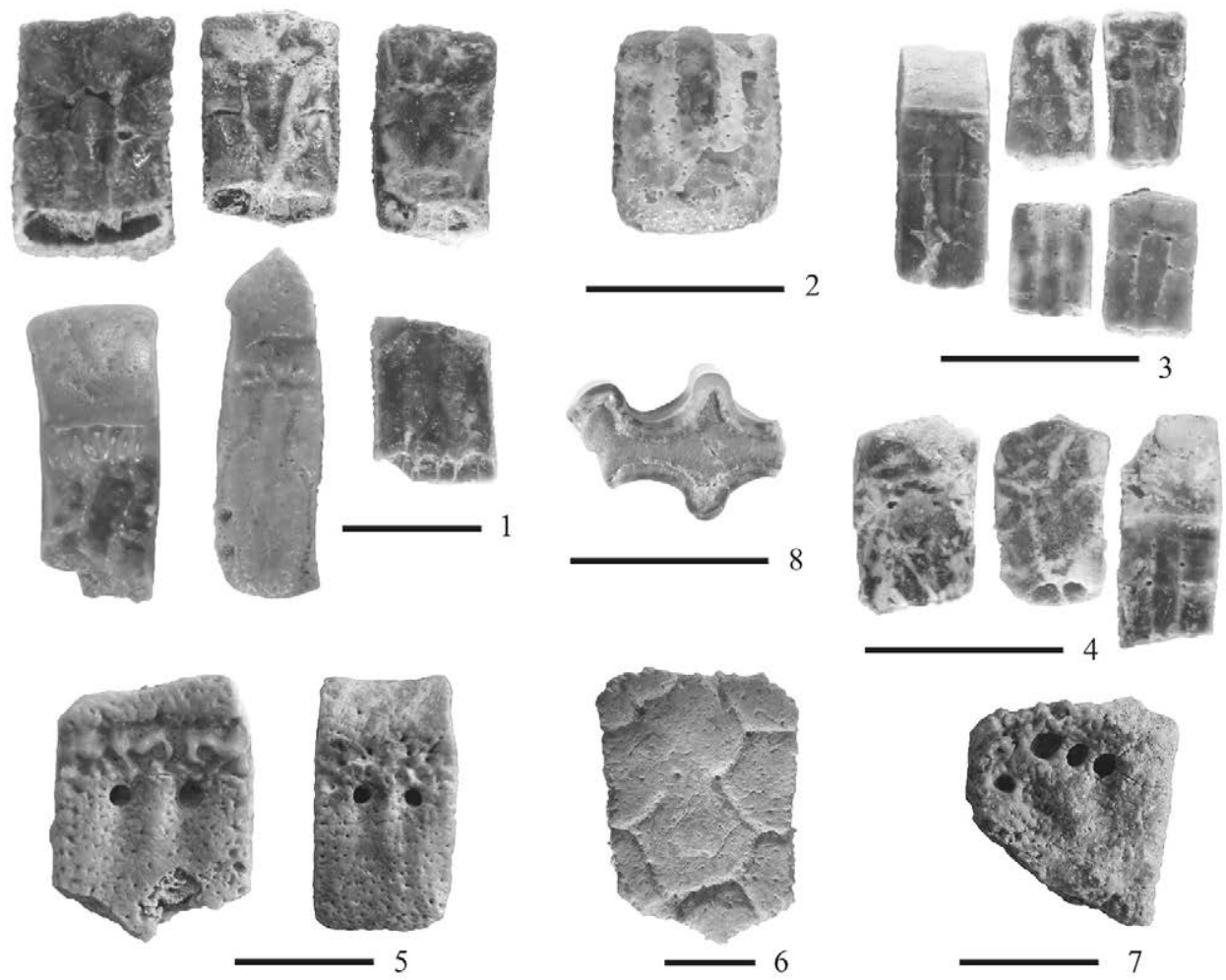

FIG. 3. Mamíferos fósiles de la localidad Barrancas Blancas. 1. Meteutatus percarinatus, MPEF-PV 2546/1-6; 2. Archaeutatus sp., MPEF-PV 2547; 3. Parutaetus cf. P. chilensis, MPEF-PV 2548/1-5; 4. Prozaedyus sp., MPEF-PV 2549/1-3; 5. Peltephilus undulatus, MPEF-PV 2550/1-2; 6. Glyptatelinae indet., MPEF-PV 2551/1-2; 7. Machlydotherium ater, MPEF-PV 2552; 8. Pseudoglyptodon cf. P. chilensis, MPEF-PV 2553, vista oclusal. Escala=1 cm. 


\section{Tribu EUPHRACTINI Winge, 1923 \\ Género Archaeutatus Ameghino, 1902}

Especie tipo: Archaeutatus malaspinensis

Ameghino, 1902

Archaeutatus sp.

Figura 3.2

Material referido: MPEF-PV 2547, una porción de osteodermo móvil (aproximadamente tercio posterior). Descripción: Osteodermo de gran tamaño y proporcionalmente delgado (la porción mide $10,7 \mathrm{~mm}$ de largo, 8,6 $\mathrm{mm}$ de ancho y 4,5 $\mathrm{mm}$ de espesor), Archaeutatus es uno de los euphractinos de mayor talla corporal en el Paleógeno. Sobre la superficie expuesta se observa la porción posterior de una figura principal lageniforme que ocupa todo el margen posterior del osteodermo. Sobre la porción anterior del fragmento conservado se observan un par de forámenes circulares (1-1,5 mm de diámetro), ubicados sobre los surcos que delimitan la figura principal al nivel donde esta se estrecha formando el 'cuello' de la figura; esto se observa típicamente en los osteodermos móviles de Archaeutatus. Sobre el margen posterior hay unos 15 forámenes pilíferos pequeños; esta es la principal característica que nos impide asignarlo a la única especie conocida del género $A$. malaspinensis, la cual posee de dos a cuatro forámenes pilíferos circulares (Ciancio y Carlini, 2008).

Comentarios: Archaeutatus malaspinensis Ameghino, 1902 es un taxón característico de la EM Deseadense, se ha mencionado para la localidad tipo de esta edad, La Flecha (Carlini et al., 2009). Por otra parte, también fue mencionado Archaeutatus aff. A. malaspinensis para los niveles de La Cantera (Gran Barranca), y una nueva especie todavía no descrita formalmente, presente en las faunas del APS de Cañadón Blanco, Lomas Blancas (=La Curandera) y Laguna de los Machos (Carlini et al., 2009 y 2010).

\section{Género Parutaetus Ameghino, 1902}

Especie tipo: Parutaetus chicoensis Ameghino, 1902 Parutaetus cf. P. chilensis Carlini, Ciancio, Flynn, Scillato-Yané y Wyss, 2009

Figura 3.3

Material referido: MPEF-PV 2548/1-5, cinco osteodermos no asociados.

Descripción: Osteodermos pequeños; las áreas de contacto entre osteodermos contiguos son cóncavas en sección, de superficie lisa y con algunas proyecciones denticulares esparcidas irregularmente. Se observa una figura principal alargada, más ancha anteriormente y sin llegar a contactar el borde posterior del osteodermo, rodeada por un par de figuras anteriores, un par de laterales anteriores y un par de laterales posteriores. La superficie externa de los osteodermos tiene cuatro perforaciones pequeñas ubicadas en la intersección del surco que delimita la figura principal con los surcos que delimitan las figuras laterales. Las características mencionadas hasta aquí permiten asignarlos a Parutaetus, pero no permiten hacer una diferenciación a nivel específico. El sistema pilífero está restringido al borde posterior y presenta dos forámenes pequeños, al igual que en P. chilensis (Carlini et al., 2009). Los osteodermos móviles tienen dos surcos longitudinales que dividen su superficie expuesta en una figura central y dos laterales que, a su vez, están divididas por un surco transversal poco profundo en el tercio anterior, que define una figura lateral anterior y otra posterior más larga.

Comentarios: P. chilensis es una de las dos especies de dasipódidos presentes en los niveles tinguiririquenses de Tinguiririca (Chile), las otras especies de Parutaetus se encuentran frecuentemente en sedimentos predeseadenses (Ameghino, 1902; Carlini et al., 2009).

\section{Género Prozaedyus Ameghino, 1891}

Especie tipo: Prozaedyus proximus Ameghino, 1887 Prozaedyus sp. Figura 3.4

Material referido: MPEF-PV 2549/1-3: tres osteodermos.

Descripción: Se describen varios osteodermos que son de pequeño tamaño, con una figura central alargada, rodeada por numerosas figuras menores y escasos forámenes pilíferos pequeños sobre el margen posterior. Estas características permiten asignarlos a Prozaedyus. Sin embargo, los restos son insuficientes para realizar la asignación específica, de acuerdo con los ejemplares tipo de las especies conocidas y probablemente correspondan a más de una especie. Comentarios: Este taxón se conoce desde la EM Deseadense en Patagonia y sus últimos registros son en el Mioceno medio. Los osteodermos que se describen aquí presentan una ornamentación primitiva, respecto a las otras especies de Prozaedyus (e.g., la 
figura central no contacta con el borde posterior, las figuras periféricas posteriores son más largas que las periféricas laterales y anteriores) similar a la de las formas presantacrucenses, como Prozaedyus humilis (Carlini et al., 2010; Kramarz et al., 2010).

\section{Familia PELTEPHILIDAE Ameghino, 1894b Género Peltephilus Ameghino, 1887 \\ Especie tipo: Peltephilus pumilus Ameghino, 1887 Peltephilus undulatus Ameghino, 1897}

Figura 3.5

Material referido: MPEF-PV 2550/1-2: dos osteodermos móviles.

Descripción: Los osteodermos móviles son anchos y relativamente delgados, poseen una superficie externa con numerosas puntuaciones que le dan un aspecto rugoso, sin figuras diferenciadas y poseen en la porción anterior del osteodermo un par de forámenes de gran tamaño. Estas características son diagnósticas de Peltephilus. La porción anterior (tecla) es corta y está separada de la porción principal del osteodermo por una zona de transición muy rugosa. Teniendo en cuenta las medidas del osteodermo más completo (19 $\mathrm{mm}$ de longitud y $10,5 \mathrm{~mm}$ de ancho), los restos podrían referirse a Peltephilus depressus o P. undulatus. Sin embargo, de acuerdo con las descripciones originales de Ameghino (1897), $P$. undulatus se define por la presencia de una carena medial ancha y poco elevada ( $P$. depressus no posee estas elevaciones) y por los dos grandes forámenes anteriores en su superficie externa $(P$. depressus posee típicamente 4 grandes forámenes).

Comentarios: Este es un taxón muy frecuente en sedimentos con fauna Deseadense; está presente en las faunas típicas de esta edad como La Flecha y Cabeza Blanca (Loomis, 1914; Scillato-Yané, 1986; Ciancio, 2010).

Familia GLYPTODONTIDAE Gray, 1869

Subfamilia GLYPTATELINAE Castellanos, 1932 Glyptatelinae indet.

Figura 3.6

Material referido: MPEF-PV 2551/1-2: un osteodermo de la coraza dorsal y un fragmento de osteodermo.

Descripción: El osteodermo tiene un contorno rectangular, y los surcos que delimitan las figuras de la cara externa son amplios y poco marcados. Posee una figura central de forma hexagonal, no circular como se observa en los Propalaehoplophorinae, Hoplophorinae (=Sclerocalyptinae) y Glyptodontinae; además esta figura está desplazada hacia el margen posterior, como es característico en los Glyptatelinae (ver Croft et al., 2007). Rodeando la figura central hay seis figuras periféricas; adicionalmente hay un par de figuras accesorias ubicadas en los vértices anterolaterales del osteodermo. En la porción anterior de la figura central, se observa un par de forámenes situados en la intersección de los surcos radiales que delimitan la figura anterior con el surco que demarca la figura central. Además, hay otro par de forámenes de la cara expuesta ubicados en los laterales de la figura anterior, entre esta y las figuras accesorias. Comentarios: Las características del osteodermo difieren de las otras especies conocidas para la subfamilia. Es posible que esté representando un nuevo taxón; sin embargo, los restos son insuficientes para poder determinarlo. Los gliptatelinos tienen sus primeros registros en el Eoceno tardío y su presencia en Patagonia se registra hasta el Oligoceno tardío (EM Deseadense).

\section{CINGULATA incertae sedis Género Machlydotherium Ameghino, 1902 Especie tipo: Machlydotherium asperum Ameghino, 1902 \\ Machlydotherium ater Ameghino, 1902 Figura 3.7}

Material referido: MPEF-PV 2552: un osteodermo fijo incompleto.

Descripción: Si bien el osteodermo está incompleto, sus características particulares permiten asignarlo específicamente. La superficie del osteodermo es muy rugosa y no presenta figuras diferenciadas, aunque posee una carena central ancha y baja. Sobre el margen anterior de la carena medial se observan cuatro forámenes de gran tamaño (2-3 mm de diámetro) dispuestos asimétricamente. Entre las especies conocidas del género, esta última característica se observa solo en M. ater (Ameghino, 1902:53).

Comentarios: Esta especie ha sido descrita por Ameghino (1902) para la EM Mustersense; Carlini et al. (2005) mencionan la presencia de Machlydotherium sp. en los niveles de 'La Cancha' en Gran Barranca, asignados temporalmente a la EM Tinguiririquense. Por otra parte, Bond et al. (1998) reconocieron la presencia de Machlydoterium sp. 
en los afloramientos de la Formación Fray Bentos (asignados a la EM Deseadense) en la provincia de Corrientes, Argentina. Sin embargo, del reestudio de esos materiales, Carlini et al. (2007) plantean que la porción de osteodermo referida a Machlydoterium sp. pertenece a un Peltephilidae indeterminado.

\section{Orden PHYLLOPHAGA Owen, 1842 Género Pseudoglyptodon Engelmann, 1987 \\ Especie tipo: Pseudoglyptodon sallaensis Engelmann, 1987 \\ Pseudoglyptodon cf. P. chilensis McKenna, Wyss y Flynn, 2006 \\ Figura 3.8}

Material referido: MPEF-PV 2553: fragmento de diente.

Descripción: Es un fragmento de diente trilobulado ( ${ }^{\text {er }}$ molariforme?), mostrando el patrón de Pseudoglyptodon (Pujos y de Iulis, 2007). En vista oclusal, se distingue un lóbulo medial comprimido mesiodistalmente y un lóbulo lateral (mesial o distal). La superficie de desgaste es plana y presenta una pared externa de una dentina más dura, la cual es más delgada que en $P$. sallaensis. Al igual que en $P$. chilensis, los surcos que separan los lóbulos son más amplios que en $P$. sallaensis. El tamaño concuerda con la medida de los dientes de $P$. chilensis.

Comentarios: Se asigna este resto con dudas a Pseudoglyptodon chilensis de acuerdo con las dimensiones y la morfología de la lobulación presente en los molariformes del material tipo de esta especie proveniente de Tinguiririca (Chile) (ver McKenna et al., 2006). Clásicamente varios autores (e.g., Ameghino, 1902; Simpson, 1948) habían asignado este tipo de dientes (trilobulados, con una capa de dentina más dura externa y una dentina más blanda ubicada en el interior) como pertenecientes a los Glyptatelinae, sin embargo, hasta ahora no se ha encontrado la evidencia suficiente para establecer si los osteodermos y los dientes definen a un mismo taxón (McKenna et al., 2006).

Orden NOTOUNGULATA Roth, 1903 Suborden TOXODONTIA Owen, 1853 Familia 'NOTOHIPPIDAE' Ameghino, 1894b Género Rhynchippus Ameghino, 1897

Especie tipo: Rhynchippus equinus Ameghino, 1897 Rhynchippus pumilus Ameghino, 1897 Figura 4.1-2
Material referido: MPEF-PV 2558/1-2: P2 izquierdo y $\mathrm{P} 4$ izquierdo con fragmento de paladar. Descripción: El P2 (MD: 7,64 mm; LL: 7,74 mm) es de forma redondeada y presenta en la superficie oclusal una delgada foseta central que se extiende anteriormente e interrumpe lingualmente el protolofo. El paracono forma una suave ondulación en el ectolofo. El P4 (MD: 9,38 mm; LL: 7,87 mm) es de aspecto trapezoidal con una cara lingual más corta que la labial. Presenta el protolofo y el metalofo bien desarrollados uniéndose en la cara lingual, encerrando una foseta alargada. El borde labial de esta foseta lleva pequeñas crestas o cúspulas, sin definir una crista o crochet. El parastilo es más estrecho que la columna del paracono y el hipocono se proyecta posterolingualmente formando un vértice.

Comentarios: Se asigna este material a Rhynchippus pumilus por sus afinidades morfológicas y métricas con el ejemplar tipo de la especie (MACN-A-5261) y con el ejemplar AMNH 29579, figurado por Chaffee (1952) como R. pumilus. Dentro del género Rhynchippus se reconocen tres especies: $R$. equinus, $R$. pumilus y $R$. brasiliensis (Cerdeño y Vera, 2010). Las diferencias entre las mismas son escasas y en el caso de $R$. pumilus, Ameghino (1897) la distinguió de $R$. equinus por su menor tamaño.

Los 'Notohippidae' se registran desde niveles referibles a la EM Casamayorense hasta el 'Piso Notohippidense' de la EM Santacrucense (López et al., 2010). El grupo ha sido usualmente separado en dos subfamilias, 'Rhynchippinae' y 'Notohippinae' (Simpson, 1945). Shockey (1997) puso en duda la validez de estos agrupamientos y a partir de su análisis filogenético los consideró como grupos parafiléticos, situación también corroborada recientemente por Marani (2005), Cerdeño y Vera (2010) y Shockey et al. (2012).

La mayor diversidad de los notohípidos ha sido registrada en las localidades deseadenses de $\mathrm{La}$ Flecha, Cabeza Blanca y Scarritt Pocket con los géneros Rhynchippus, Morphippus y Eurygenium. Recientemente se han descrito nuevos taxones de notohípidos para la localidad de Gran Barranca en Patagonia (Marani y Dozo, 2008; López et al., 2010) y varios notohípidos de esta edad también se mencionan en localidades deseadenses fuera de Patagonia (Shockey, 1997; Shockey et al., 2009; Cerdeño y Vera, 2010). 

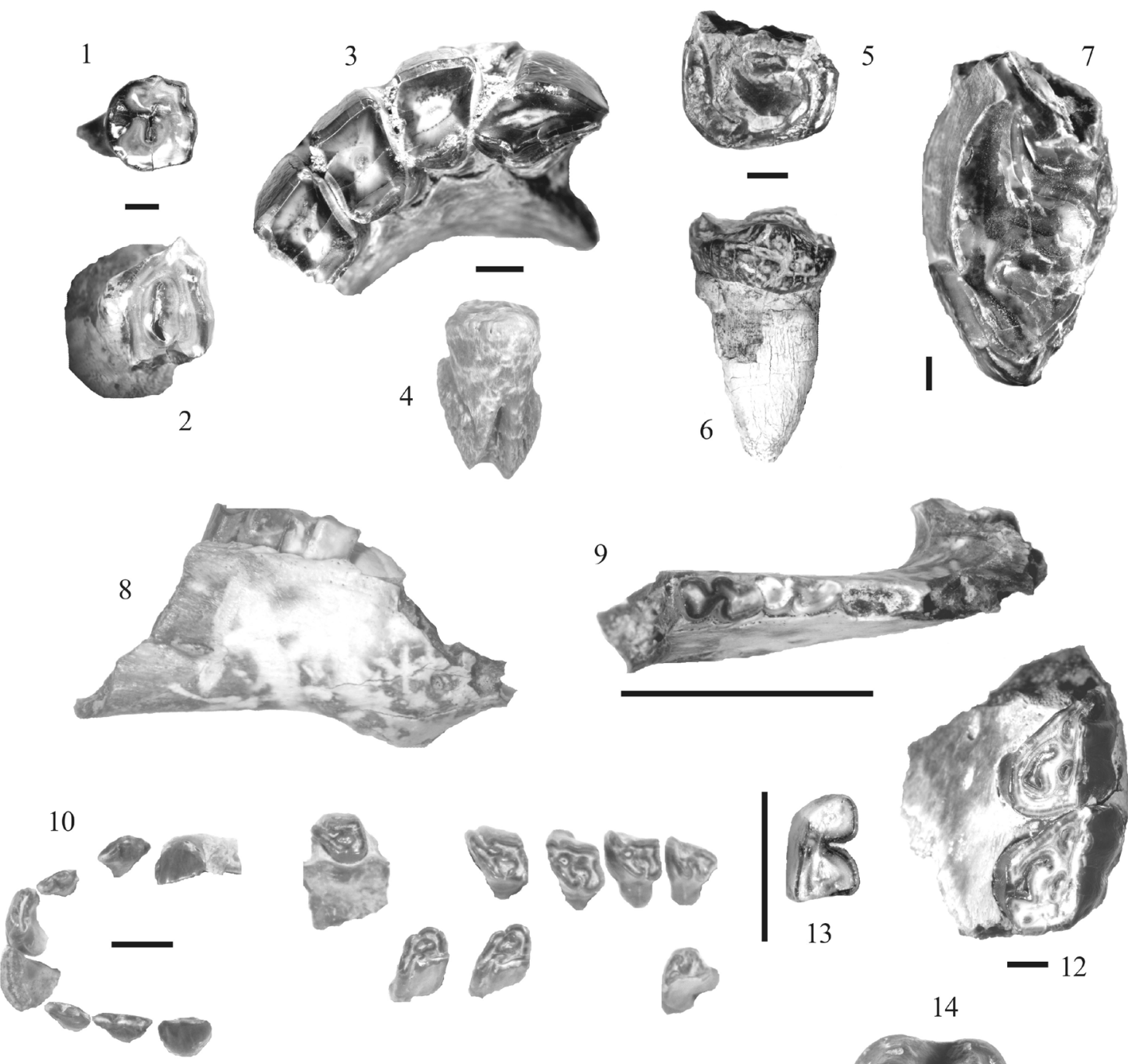

14
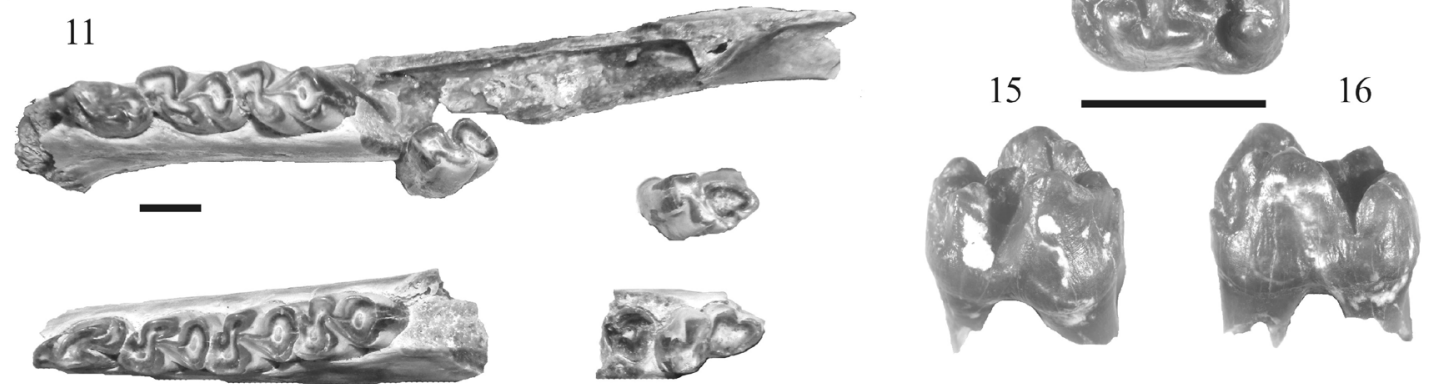

FIG. 4. Mamíferos fósiles de la localidad Barrancas Blancas. 1-2. Rhynchippus pumilus, MPEF-PV 2558/1-2, vista oclusal; 3-4. 'Notohippidae' indet.; 3. MPEF-PV 2554, vista oclusal; 4. MPEF-PV 2556, vista dorsal; 5-6. Leontinia gaudryi?, MPEF-PV 2559; 5. vista oclusal; 6. vista labial; 7. Isotemnidae indet., MPEF-PV 2562, vista oclusal; 8-9. Eopachyrucos pliciferus, MPEF-PV 2564, 8. vista labial, 9. vista oclusal; 10-11. Proargyrohyrax curanderensis, MPEF-PV 2565, vista oclusal; 12. Pseudhyrax eutrachytheroides?, MPEF-PV 2568, vista oclusal; 13. Pachyrukhinae indet., MPEF-PV 2570, vista oclusal; 14-16. Proterotheriidae indet., MPEF-PV 2571; 14. vista oclusal; 15. vista labial; 16. vista lingual. Escala=1 cm. 


\section{'Notohippidae' indet. Figura 4.3-4}

Material referido: MPEF-PV 2554, fragmento de rama mandibular derecha con i1-3 y c; MPEF-PV 2555/1-3: dos $m 2$ derechos aislados y un fragmento de $\mathrm{m} 1$ izquierdo aislado; MPEF-PV 2556: una falange ungueal; MPEF-PV 2557/1-2: dos M2 izquierdos aislados, MPEF-PV 2557/3: un I3 derecho aislado, MPEF-PV 2557/4: un C derecho aislado.

Descripción: El fragmento de la rama mandibular permite inferir una arcada redondeada; al igual que en Rhynchippus equinus y Eurygenium pacegnum, los caninos están contenidos dentro de la arcada. Los incisivos presentan la cara labial convexa y el canino es incisiviforme, pero algo alargado en sentido mesiodistal, como en Rhynchippus equinus. Los molares inferiores, como se observa en representantes del género Rhynchippus, presentan un trigónido más corto que el talónido y dos fosétidas, una entre el trigónido y el talónido y otra en el entolófido. La falange ungueal al igual que en otros 'Rhynchippinae' (Eurygeniuum, Rhynchippus, Moqueguahippus), tiene una fisura longitudinal distal (Shockey et al., 2009).

Comentarios: En principio estos materiales, por su mayor tamaño, no corresponden a la especie Rhynchippus pumilus, pero son insuficientes para poder realizar una determinación más precisa a nivel genérico y/o específico.

\section{Familia LEONTINIIDAE Ameghino, 1894a Género Leontinia Ameghino, 1894a \\ Especie tipo: Leontinia gaudryi, Ameghino, 1894a Leontinia gaudryi? Ameghino, 1894a Figura 4.5-6}

Material referido: MPEF-PV 2559: P1 derecho incompleto; MPEF-PV 2560: I1 izquierdo; MPEF-PV 2561/1-2: dos C derechos.

Descripción: El P1 (MD: 13,59 mm) está incompleto, faltándole casi $50 \%$ de su pared labial. Como se observa en el ejemplar de Leontinia gaudryi que describe Patterson (1934, Fig. 10), el conspicuo protocono se conecta por medio de dos cíngulos al parastilo y al centro de la cara posterior del diente, donde se observa un metacónulo. El incisivo corresponde al I1 (MD: 10,29 mm; LL: 13,80) y guarda afinidad con los caracteres que mencionan Shockey et al. (2012) para L. gaudryi. En ese sentido es in- cisiviforme, presenta cierta compresión lateral y la superficie oclusal es plana y triangular. Los caninos (1-MD: $14.94 \mathrm{~mm}$; LL: 13,38 mm; 2-MD: 16,08; LL: 13,52) presentan un cíngulo labial arqueado y un cíngulo lingual desarrollado. Son de contorno triangular en vista lingual. Las medidas de los dientes descritos se corresponden con algunos de los ejemplares figurados por Chaffee (1952) como L. gaudryi.

Comentarios: Estos materiales se asignan con dudas a Leontinia gaudryi porque, aunque comparten caracteres morfológicos con ejemplares que tanto Patterson (1934) como Chaffee (1952) refieren a esta especie, existen diferencias en los caracteres métricos. En particular, las medidas del P1 son menores que las dadas en general para Leontinia y Scarritia.

Los Leontiniidae son Toxodontia de mediano a gran tamaño, característicos de América del Sur, entre el Eoceno tardío (EM Mustersense) y el Mioceno medio (EM Laventense). Durante la EM Deseadense alcanzan su mayor diversidad, sobre todo en la parte sur de América del Sur (Shockey et al., 2012). Sus caracteres más destacables incluyen incisivos caniniformes y una dentición braquiodonta a mesodonta. La distribución de la especie Leontinia gaudryi está restringida a la parte norte de la provincia de Santa Cruz (La Flecha, Cerro Alto, Piedra Negra, Monte Espejo, Estancia 8 de Julio) y en el centro de Chubut (Cabeza Blanca y Las Cascadas) (Ribeiro et al., 2010).

\section{Familia ISOTEMNIDAE Ameghino, 1897 Isotemnidae indet. Figura 4.7}

Material referido: MPEF-PV 2562: m3 izquierdo incompleto.

Descripción: El m3 (MD: ca. 27 mm; LL: $11 \mathrm{~mm}$ ) está incompleto faltándole parte del trigónido a la altura del hipofléxido. Como se observa en la descripción del género Pleurostylodon (Simpson, 1967), el m3 presenta un talónido alargado con un prominente hipoconúlido separado como un lóbulo proyectado posteriormente.

Comentarios: El m3 está incompleto, pero por su morfología, que da cuenta de un corto trigónido, puede referirse a la Familia Isotemnidae. En particular para la EM Deseadense, Ameghino (1895) da a conocer, a partir de dientes sueltos y una mandíbula incompleta, los géneros Pleurocoelodon y Trimerostephanos, 
pero por lo fragmentario del material presentado es difícil asignarlo a alguno de estos géneros.

Los Isotemnidae son Toxodontia primitivos, braquiodontes que aparecen en Patagonia en el Paleoceno tardío (EM Riochiquense), se constituyen en un grupo muy diverso durante el Eoceno, y su último registro es en la EM Deseadense (Marshall et al., 1983). A juzgar por el tamaño dentario, son los más grandes notoungulados durante las EM Casamayorense y Mustersense (Madden, 1990). Se los registra también en Tinguiririca, región central de Chile (EM Tinguiririquense), en otras localidades predeseadenses de Patagonia (Croft et al., 2008), en la Formación Fray Bentos del Deseadense de Uruguay (Ubilla et al., 1999) y en el Eoceno de Jujuy (Argentina) (Bond y López, 1995).

Familia INTERATHERIIDAE Ameghino, 1887 Subfamilia INTERATHERIINAE Simpson, 1945 Género Eopachyrucos Ameghino, 1901 Especie tipo: Eopachyrucos pliciformis Ameghino, 1901

Eopachyrucos pliciferus Ameghino, 1901 Figura 4.8-9

Material referido: MPEF-PV 2564, mandíbula incompleta, con el sector de la sínfisis, alvéolos de los i, c y p1 derechos y p2-3 derechos.

Descripción: El p2 (MD: 3,7 mm; LL: 1,6 mm) es alargado y con una configuración trilobulada. El trigónido está desarrollado con un protocónido conspicuo y el margen anteroexterno elevado. El metacónido está proyectado lingualmente y la metacrístida, orientada transversalmente, conecta el protocónido con el metacónido. El talónido, pequeño y redondeado, está limitado a los lados por dos surcos y conecta directamente al metacónido amplio por un istmo delgado. El p3 (MD: 3,8 mm; LL: $2 \mathrm{~mm}$ ) es molariforme, con un trigónido más grande que el talónido. A la altura del metacónido hay una conexión con el talónido redondeado.

Comentarios: Este ejemplar se asigna a Eopachyrucos pliciferus porque comparte caracteres morfológicos y métricos con los ejemplares MLP 12-1529 y MLP 91-IX-5-17 que Reguero (1999) y Hitz et al. (2000) refieren a dicha especie. En ese sentido y a pesar de lo fragmentario del material, se destacan varios de los caracteres diagnósticos tales como premolares con coronas altas y surco anterointerno del trigónido persistente. Además se observa que el talónido de los premolares, a diferencia de lo observado en Proargyrohyrax curanderensis, es más corto y menos redondeado.

Los Interatheriidae son notoungulados herbívoros de pequeño a mediano tamaño, muy bien representados en la mayor parte de las faunas del Cenozoico de América del Sur (Hitz et al., 2000). Los primeros registros de interaterinos ocurren en la fauna de Tinguiririca (EM Tinguiririquense) y en Patagonia provienen del nivel denominado 'Astraponotense más superior' (Edad Tinguiririquense) de Gran Barranca, Cañadón Blanco y Rocas Bayas (Reguero, 1993; Hitz et al., 2000; Croft et al., 2008). En particular, el holotipo de Eopachyrucos pliciferus fue colectado en los niveles 'Astraponotense más superior' (Edad Tinguiririquense) de Gran Barranca (Bond et al., 1996; Hitz et al., 2000).

\section{Género Proargyrohyrax Hitz, Reguero, Wyss y Flynn, 2000}

Especie tipo: Proargyrohyrax curanderensis Hitz, Reguero, Wyss y Flynn, 2000

\section{Proargyrohyrax curanderensis Hitz, Reguero, Wyss y Flynn, 2000 \\ Figura 4.10-11; Tabla 1}

Material referido: MPEF-PV 2565, fragmento de rama mandibular derecha con p2-p4; $\mathrm{m} 1$ o m2 derecho; fragmento mandibular izquierdo con $\mathrm{p} 2-$ $\mathrm{m} 1 ; \mathrm{m} 3$ derecho; $\mathrm{m} 3$ izquierdo; I1-3 derechos; I1-3 izquierdos; $\mathrm{C}$ derecho e izquierdo; P3 derecho; P4 derecho; M3 derecho; P2 izquierdo; P4 izquierdo; M1-3 izquierdos; MPEF-PV 2566, M1 o M2 izquierdo; MPEF-PV 2567, $\mathrm{m} 1$ o $\mathrm{m} 2$ izquierdo.

Descripción: El material del ejemplar MPEF-PV 2565 es fragmentario y la mayor parte de los dientes están aislados, pero fueron localizados en contigüidad, por lo que es muy probable que pertenezcan al mismo individuo. Las series dentarias superior e inferior se asocian por compatibilidad en el tamaño $\mathrm{y}$ en el tipo de desgaste.

El I1 es particularmente ensanchado, presenta una raíz y es de sección ovalada; el I2 es de pequeño tamaño, con una superficie coronaria arriñonada; el I3 es de mayor tamaño y ligeramente más alargado que el I2; el canino es ligeramente mayor que los I2-I3 y, al igual que los incisivos, está comprimido lateralmente y presenta una cresta vertical externa anterior. Los P2, P3 y P4 son molariformes, cuadrangulares y presentan una morfología similar, 
TABLA 1. MEDIDAS DENTARIAS DE PROARGYROHYRAX CURANDERENSIS (MM).

\begin{tabular}{|c|c|c|c|c|c|c|c|c|}
\hline \multirow{2}{*}{ Dientes } & \multirow{2}{*}{ Medidas } & \multicolumn{2}{|c|}{ MPEF-PV2565 } & \multirow{2}{*}{$\begin{array}{l}\text { MLP-61- } \\
\text { VIII-3-27 }\end{array}$} & \multirow{2}{*}{$\begin{array}{l}\text { MLP-61- } \\
\text { VIII-3-24 }\end{array}$} & \multirow{2}{*}{$\begin{array}{l}\text { MLP-61- } \\
\text { IV-14-1 }\end{array}$} & \multirow{2}{*}{$\begin{array}{l}\text { MLP-93- } \\
\text { XI-25-3 }\end{array}$} & \multirow{2}{*}{$\begin{array}{l}\text { MLP-93- } \\
\text { XI-25-19 }\end{array}$} \\
\hline & & Izq. & Der. & & & & & \\
\hline \multirow[t]{2}{*}{ I1 } & MD & 8,40 & 8,00 & - & - & - & - & - \\
\hline & LL & 1,00 & 1,00 & - & - & - & - & - \\
\hline \multirow[t]{2}{*}{$\mathrm{I} 2$} & MD & 5,00 & 5,00 & - & - & - & - & - \\
\hline & LL & 1,00 & 1,00 & - & - & - & - & - \\
\hline \multirow[t]{2}{*}{ I3 } & MD & 7,80 & 8,00 & - & - & - & - & - \\
\hline & LL & 2,00 & 2,00 & - & - & - & - & - \\
\hline \multirow[t]{2}{*}{$\mathrm{C}$} & MD & 6,90 & 6,80 & - & - & - & - & - \\
\hline & LL & - & - & - & - & - & - & - \\
\hline \multirow[t]{2}{*}{ P1 } & MD & - & - & - & - & - & - & - \\
\hline & LL & - & - & - & - & - & - & - \\
\hline \multirow[t]{2}{*}{ P2 } & MD & 6,80 & - & - & - & - & - & - \\
\hline & $\mathrm{LL}$ & 4,20 & - & - & - & - & - & - \\
\hline \multirow[t]{2}{*}{ P3 } & MD & - & 7,00 & - & - & - & - & - \\
\hline & LL & - & 6,00 & - & - & - & - & - \\
\hline \multirow[t]{2}{*}{ P4 } & MD & 7,30 & 7,30 & 7,70 & - & - & - & - \\
\hline & LL & 6,00 & 6,20 & 6,40 & - & - & - & - \\
\hline \multirow{2}{*}{ M1 } & MD & 7,50 & - & 8,10 & - & - & - & - \\
\hline & LL & 6,50 & - & 6,70 & - & - & - & - \\
\hline \multirow[t]{2}{*}{ M2 } & MD & 6,80 & - & 7,90 & - & - & - & - \\
\hline & LL & 4,80 & - & 5,60 & - & - & - & - \\
\hline \multirow[t]{2}{*}{ M3 } & MD & 6,50 & 6,50 & 6,80 & - & - & - & - \\
\hline & LL & 4,00 & 4,00 & 4,00 & - & - & - & - \\
\hline \multirow{2}{*}{$\mathrm{p} 2$} & MD & 7,80 & 7,80 & - & 7,10 & - & - & - \\
\hline & LL & 3,20 & 3,20 & - & 3,50 & - & - & - \\
\hline \multirow{2}{*}{ p3 } & MD & 7,80 & 8,00 & - & 7,50 & 7,20 & 7,40 & - \\
\hline & LL & 4,00 & 4,00 & - & 4,40 & 3,90 & 4,30 & - \\
\hline \multirow[t]{2}{*}{$\mathrm{p} 4$} & MD & 7,50 & 7,50 & - & 7,70 & 7,10 & 7,60 & - \\
\hline & LL & 4,50 & 4,50 & - & 4,50 & 4,00 & 4,40 & - \\
\hline \multirow[t]{2}{*}{$\mathrm{m} 1$} & MD & 7,00 & - & - & 7,60 & 7,30 & - & 7,40 \\
\hline & LL & 4,50 & - & - & 5,00 & 4,40 & - & 4,50 \\
\hline \multirow[t]{2}{*}{$\mathrm{m} 2$} & MD & - & 7,00 & - & 8,10 & 7,40 & - & - \\
\hline & LL & - & 4,20 & - & 4,80 & 4,10 & - & - \\
\hline \multirow[t]{2}{*}{$\mathrm{m} 3$} & MD & 7,50 & 7,50 & - & 7,70 & 8,80 & - & - \\
\hline & LL & 4,20 & 4,00 & - & 3,60 & 3,50 & - & - \\
\hline
\end{tabular}

siendo el P2 de menor tamaño. Al igual que en el holotipo de $P$. curanderensis (Hitz et al., 2000) se destaca un parastilo bien desarrollado que se curva con una orientación labial y se conecta a la columna del paracono, moderadamente desarrollada.
El paracono y el parastilo están separados por un profundo surco oblicuo y se observa una foseta labial anteroexterna. El protolofo y el metalofo demarcan una notable foseta central con forma de corazón. En los premolares, el surco lingual está ausente, aunque 
hay un ligero indicio en el P4, posiblemente por el menor grado de desgaste. Como se observa en el holotipo, los molares son más largos que anchos y están interrumpidos lingualmente por un profundo surco bífido. El M1 es el más grande de la serie y en él se destacan dos fosetas, una anteroexterna de gran tamaño cerca del paracono y otra posteroexterna más pequeña cerca del metacono. El surco lingual es delgado y se observa un pequeño lóbulo medio en la fosa principal. Posee una gruesa raíz interna y dos raíces externas fusionadas. El M2 es más estrecho que el M1, pero al igual que este tiene tres raíces. Se observa también un surco lingual y el lóbulo medio está más desarrollado que en el M1. El M3, al igual que en el holotipo, es el molar más pequeño y presenta un aspecto bilobulado. En particular, se destaca el gran desarrollo de la foseta anteroexterna.

En la serie inferior, el p2 es estrecho, presenta un trigónido y un talónido diferenciado, pero este último es considerablemente menor que el primero. El trigónido consiste en un paracónido anterior conectado por un pequeño puente al metalófido, orientado en sentido diagonal. Se observa además un delgado lófido, correspondiente al posmetastílido, conectado al metacónido del lado labial y proyectado posteriormente. También se evidencia un profundo surco entre el paracónido y el metalófido. El p3, al igual que en el holotipo, es molariforme y presenta el trigónido y el talónido ensanchados, equidimensionales y casi del tamaño de los molares. El trigónido es de aspecto cuadrangular con un surco anterointerno. Un surco labial profundo y estrecho separa el trigónido del talónido. El p4 es muy semejante al p3, pero muestra un posmetastílido menos desarrollado y menos proyectado posteriormente. El m1 es muy parecido al p4, excepto en que el trigónido es más ancho y redondeado y falta el posmetastílido de los premolares. El $\mathrm{m} 3$ presenta un talónido más grande que el trigónido, con un ligero surco posterolingual. Comentarios: Este es el primer caso en donde se dan a conocer las series dentarias superior e inferior asociadas de un mismo individuo de $P$. curanderensis.

El holotipo de esta especie proviene de la localidad Lomas Blancas, cerca de La Curandera, Departamento Paso de Indios, en la provincia del Chubut. Hitz et al. (2000) consideraron esta localidad bioestratigráficamente correlativa al 'Astraponotense más superior', y recientemente Reguero y Prevosti (2010) la consideran correlativa a la EM Tinguiririquense.
Familia 'ARCHAEOHYRACIDAE'Ameghino, 1897 Género Pseudhyrax Ameghino, 1901

Especie tipo: Pseudhyrax eutrachytheroides Ameghino, 1901

Pseudhyrax eutrachytheroides? Ameghino, 1901 Figura 4.12

Material referido: MPEF-PV 2568: fragmento de paladar con M1-2 izquierdos; MPEF-PV 2569/1-2: P2 ó P3 derecho; P2 ó P3 izquierdo.

Descripción: Los molares son protohipsodontes, de forma trapezoidal y se destaca el desarrollo alcanzado por el parastilo. El M1 (MD: 11,09 mm; LL: 8,37 mm) presenta un ectolofo sinuoso y dos fosetas labiales, una anterior y otra posterior, esta última a la altura del metacono. Se destaca una foseta central alargada, dilatada sobre todo en el extremo postero lingual. El M2 (MD: 11,83 mm; LL: 7,33 mm), más alargado que el M1, presenta también un ectolofo sinuoso y dos fosetas labiales, una anterior y otra posterior. La foseta central, de mayor desarrollo que en el M1, se abre en un surco lingual que la divide en dos ramas, la anterior más extendida que la posterior, y se destacan varias microfosetas. Una se observa en el extremo de la rama anterior de la foseta central, otra a continuación de la foseta labial posterior y otra, en el ángulo posterior del lado lingual.

Comentarios: Aunque varios de los caracteres descritos muestran afinidades con Pseudhyrax (Croft et al., 2003): prominente parastilo en el ectolofo de los molares superiores, fosetas accesorias, presencia de una foseta alargada central, orientada anterolabialmente, y presencia de un ligero surco sobre el endolofo que divide protocono del hipocono, se asigna con dudas a Pseudhyrax eutrachytheroides por lo fragmentario del material. Los Archaeohyracidae constituyen una enigmática familia de notoungulados conocidos solamente en sitios paleógenos de América del Sur (Simpson, 1967; Reguero et al., 2003; Croft et al., 2003; Billet et al., 2009). Se los considera un grupo parafilético y experimentaron una gran radiación en el Eoceno tardío u Oligoceno temprano como lo indica la gran diversidad en la fauna 'Tinguiririquense' de Chile y Argentina (Croft et al., 2003; Flynn et al., 2003; Reguero et al., 2008). Según Croft et al. (2008), Tinguiririca se constituye, hasta ahora, en la única localidad de Edad Tinguiririquense en registrar a Archaeohyracidae del género Pseudhyrax, un taxon típico de la Edad Mustersense en Patagonia. 


\section{Familia HEGETOTHERIIDAE Ameghino, 1894b Hegetotheriidae indet. Figura 4.13}

Material referido: MPEF-PV 2570: $\mathrm{m} 1 \mathrm{o} \mathrm{m} 2$ derecho. Descripción: El molar es un diente simple (MD:3,97 mm; LL: $2,35 \mathrm{~mm}$ ), con un surco labial conspicuo que delimita dos lóbulos, el anterior de menor tamaño y el posterior de contorno triangular.

Comentarios: Por lo fragmentario del ejemplar, es imposible realizar una asignación más precisa; sin embargo, por el pequeño tamaño podría corresponder a un Pachyrukhinae. El biocrón de esta subfamilia va desde la EM Tinguiririquense hasta el Plioceno tardío-Pleistoceno temprano. La mayor diversificación de este grupo se registra en la Edad Deseadense, con los géneros Prosotherium, Propachyrucos y Medistylus (Reguero et al., 2007).

\section{Orden LITOPTERNA Ameghino, 1889 Familia PROTEROTHERIIDAE Ameghino, 1887 Proterotheriidae indet. Figura 4.14-16}

Material referido: MPEF-PV 2571: $\mathrm{p} 4 \mathrm{o} \mathrm{m} 1$ derecho. Descripción: El diente es de aspecto bunoselenodonte con una distancia mesiodistal mayor que la labio-lingual (MD: 12,50 mm; LL: 8,21 mm). El trigónido es ligeramente más ancho que el talónido con un hipofléxido muy marcado. En el trigónido, la cúspide más voluminosa es el metacónido; le sigue en tamaño el protocónido y a la misma altura pero por delante del metacónido se observa un pequeño paracónido. Detrás del protocónido se advierte la protocrístida posteromedial. A nivel de la paracrístida, en la región anteromedial del diente, se observa una pequeña cúspide. En el talónido, se destaca el hipocónido, de gran tamaño y se observa el entocónido mayor que el hipoconúlido contiguo. Se advierte también un mesocónido sobre la crístida oblicua. En el perímetro del diente se distingue un precingúlido conspicuo, en la región anterolabial, y un postcingúlido en la parte posterolabial.

Comentarios: Este ejemplar corresponde a un molariforme bunoselenodonte correspondiente a un Proterotheriidae primitivo. El material es fragmentario, pero comparte algunos caracteres diagnósticos de la especie Lambdaconus suinus Ameghino, 1897, como el entocónido desarrollado y su mayor bunodoncia. Según Soria (2001), esta especie corresponde al
Proterotheriidae mejor representado de la Edad Deseadense.

\section{Discusión y Conclusiones}

\subsection{Localización estratigráfica de la secuencia}

Los primeros trabajos geológicos realizados en la zona de Camarones (Chubut) fueron los relevamientos efectuados por Flores (1956) quien menciona varias unidades estratigráficas, entre ellas el Sarmientense que correspondería a las sedimentitas continentales de la Formación Sarmiento. Camacho (1979), posteriormente, no incluyó esta unidad en el cuadro estratigráfico de la Hoja Bahía Camarones. En cambio, Lema et al. (2001), en la nueva Hoja Geológica de Camarones, señalan que los afloramientos de la Formación Sarmiento (Eoceno medio-Mioceno temprano) están constituidos por material epipiroclástico y se asientan en discordancia erosiva sobre el Complejo Marifil, de edad jurásica, la Formación Salamanca, de origen marino, y los sedimentos continentales de la Formación Río Chico, estas dos últimas de edad paleocena.

La Formación Sarmiento, que subyace a las sedimentitas marinas de la Formación Chenque, es de distribución saltuaria y se halla mejor representada en el sector nororiental de la Hoja, extendiéndose en la parte austral hasta el sur de las Estancias La Ernesta y El Sauce, donde desaparecen bajo los cordones litorales y terrazas marinas.

Las denominadas Tobas de Sarmiento (Feruglio, 1949), Grupo Sarmiento (Andreis et al., 1975; Spalletti y Mazzoni, 1977) o Formación Sarmiento (Lema et al., 2001), de edad del Eoceno medioMioceno temprano, además de representar una de las unidades potencialmente más expresivas de los eventos geológicos del Paleógeno de la Patagonia extrandina, constituye una de las unidades más representativas de esa región como portadora de abundantes mamíferos fósiles, los que conforman una parte de la sucesión estándar de los vertebrados cenozoicos sudamericanos. Así se han podido reconocer varias Edades-mamífero (EMs), incluidas algunas subedades: Subedades 'Barranquense' y 'Vaquense' de la EM Casamayorense, EM Mustersense, EM Tinguiririquense (originalmente formalizada en Chile Central, Flynn et al., 2003), EM Deseadense, EM Colhuehuapense y Subedad 'Pinturense' de la EM Santacrucense (Pascual et al., 
1965; Pascual y Odreman Rivas, 1973; Pascual et al., 1996, Ré et al., 2010).

Los depósitos de la Formación Sarmiento habrían sido el resultado de la acumulación eólica de material fino piroclástico (paleoloess) en un ambiente de llanuras y durante su depositación habrían imperado condiciones climáticas cálido-templadas con la existencia de períodos estacionales húmedos relativamente extensos que alternaban con otros cortos de mayor sequedad ambiental (Andreis et al., 1975; Spalletti y Mazzoni, 1977).

\subsection{Implicancias biocronológicas y paleobio- geográficas}

Algunos datos inéditos aportados por Mario Franchi, de la Secretaría de Minería, quien junto a otros geólogos de dicha institución desarrollaron la Hoja Geológica de Camarones (Lema et al., 2001), indicaban prima facie que la localidad del Paleógeno 'Barrancas Blancas' sería de la EM Deseadense por la presencia de un ejemplar asignado con dudas al género Plagiarthrus.

Los nuevos hallazgos paleontológicos, más numerosos y diversos, confirmarían en principio la EM Deseadense para estos niveles. En este sentido, los géneros Leontinia? y Rhynchippus son considerados por Marshall et al. (1983) como fósiles guías de la EM Deseadense. Sin embargo, el panorama es algo más complejo, ya que varios taxones de xenartros y notoungulados sugieren una EM Tinguiririquense y también un intervalo pos-Tinguiririquense a preDeseadense.

En una primera aproximación de la fauna de xenartros se pudieron reconocer los siguientes taxones: Prozaedyus sp.; Sadypus sp.; Stenotatus sp.; Peltephilidae indet.; Pampatheriidae? indet.; Glyptodontidae, Glyptatelinae indet. (Dozo et al., 2004). Sin embargo, sobre la base de recientes estudios acerca de la fauna de Cingulata de otros sitios del Paleógeno de Patagonia (Carlini et al., 2004, 2005, 2009, 2010; Ciancio y Carlini, 2008), ha sido posible actualizar y ajustar las asignaciones taxonómicas de la fauna presente en Barrancas Blancas (ver arriba) y su correlación con otros yacimientos. Esta fauna no representa una asociación típica de la EM Deseadense (Oligoceno tardío), tal como se registra en 'La Flecha', Santa Cruz, Argentina (Localidad tipo de la EM Deseadense) o en 'Cabeza Blanca', Chubut, Argentina (Ciancio, 2010).
El ejemplar asignado a Archaeutatus sp. está presente en localidades referidas a la EM Tinguiririquense (Carlini et al., 2009; Ciancio, 2010). Carlini et al. (2009) describen a Parutaetus chilensis (Euphractini) y Barrancatatus tinguiririquensis (Eutatini) provenientes de Tinguiririca (Chile), localidad tipo de la EM Tinguiririquense. Estos taxones no son frecuentes en los yacimientos de edades equivalentes en Patagonia. En Barrancas Blancas, se encuentran osteodermos que se asignan a Parutaetus cf. P. chilensis, ya que se observan algunas diferencias con el material tipo de la especie. Esta especie también se halla en Gran Barranca (Chubut), en los niveles denominados 'La Cancha' -asignados a la EM Tinguiririquense (Kay et al., 1999; Ré et al., 2010; Carlini et al., 2010)- y con dudas en los niveles de 'La Cantera' (Oligoceno inferior). La otra especie descrita para la localidad tipo del Tinguiririquense no se ha registrado en Barrancas Blancas ni en otros yacimientos de Patagonia.

Una situación que llama la atención en Barrancas Blancas es la escasez de eutatinos, ya que son especialmente diversos en yacimientos del Oligoceno de Patagonia, especialmente en los niveles Tinguiriquenses de Gran Barranca (Carlini et al., 2004, 2005, 2010; Ciancio y Carlini, 2007). El único Eutatini que se registra es Meteutatus percarinatus; este taxón también está presente en Gran Barranca en los niveles correspondientes a 'La Cancha', en donde han sido identificados como Meteutatus sp. (Carlini et al., 2010).

Se ha registrado una especie de Peltephilidae, que hemos asignado a Peltephilus undulatus, descrita por Ameghino (1897) para la EM Deseadense de Patagonia. Si bien los Peltephilidae son frecuentes a partir de niveles Deseadenses, hay registros de Peltephilidae en niveles inferiores en otras localidades de Patagonia (Carlini et al., 2005; Scillato-Yané, 1986). Machlydotherium ater ha sido descrito por Ameghino (1902) como un taxón presente en niveles correspondientes a la EM Mustersense en Patagonia.

El único gliptodonte registrado corresponde a Glyptatelinae indet. y, si bien los Glyptatelinae están representados en Patagonia en el lapso MustersenseDeseadense, estos restos no pueden asignarse a ninguno de los Gliptatelinos conocidos hasta el momento, aunque comparten similitudes con un ejemplar colectado en depósitos de la Formación Fray Bentos, en Uruguay, asignados a la EM Deseadense, presentado originalmente en Bostelmann et al. (2011). 
Finalmente, hay que destacar la presencia de un molar que hemos asignado a Pseudoglyptodon $\mathrm{cf}$. chilensis, taxón presente en los sedimentos de la localidad tipo de la EM Tinguiririquense (Flynn et al., 2003; McKenna et al., 2006). Si se confirma su asignación específica, este sería el primer registro de este taxón fuera de la localidad tipo.

En cuanto a los notoungulados Isotemnidae, característicos durante el Eoceno, su último registro es en la EM Deseadense y se los registra también en Tinguiririca y en otras localidades pre-Deseadenses de Patagonia (Croft et al., 2008).

Los Interatheriinae se registran por primera vez en la EM Tinguiririquense en su localidad tipo (Flynn et al., 2003). En Patagonia, los primeros registros de interaterinos provienen de niveles de Edad Tinguiririquense de Gran Barranca, Cañadón Blanco y Rocas Bayas (Reguero, 1993; Hitz et al., 2000; Croft et al., 2008).

Reguero (1999) y Reguero et al. (2003) consideran que la especie Eopachyrucos pliciferus es indicadora del intervalo pos-Mustersense a pre-Deseadense. Según Reguero (1999) y Hitz et al. (2000), Ameghino (1901) creó este taxón a partir de un molar superior hallado en 'Gran Barranca' en probables niveles de EM Tinguiririquense de la Formación Sarmiento. Además de la localidad tipo, E. pliciferus es conocido en las localidades Lomas Blancas (La Curandera) y Cañadón Blanco (Chubut) y Rocas Bayas (Río Negro) también de EM Tinguiririquense.

Proargyrohyrax curanderensis es un taxón típico del Tinguiririquense (Hitz et al, 2000; Croft et al., 2008; Reguero y Prevosti, 2010).

En cuanto al ejemplar asignado tentativamente al género Pseudhyrax?, dicho taxón que era conocido solo en niveles de la EM Mustersense y es considerado como fósil guía para esa edad (Marshall et al., 1983), también es mencionado en la asociación faunística de la localidad de Tinguiririca en Chile central y correspondería a su última aparición (Flynn et al., 2003). Reguero (1999) también menciona este género para algunas localidades de Patagonia con fauna del Tinguiririquense, tal es el caso de la localidad Lomas Blancas (La Curandera) en el departamento Paso de Indios en el Chubut.

La localidad de Barrancas Blancas en el SE de la provincia de Chubut ofrece, al igual que el sitio de Tinguiririca y otros sitios relacionados de Patagonia, la coexistencia de taxa característicos de la EM Deseadense (e.g., Rhynchippus, Leontinia?) y taxa tradicionalmente conocidos en la EM Mustersense (Pseudhyrax?).

En síntesis, la diversidad de xenartros y de varios grupos de tipoterios (Interatheriidae y 'Archaeohyracidae') presentes en Barrancas Blancas podría corresponder a una fauna de EM Tinguiriquense o incluso podría estar representando una fauna algo más moderna, equivalente a los niveles de La Cantera (Gran Barranca). La Cantera posee dataciones de ca. $30 \mathrm{Ma}$, lo que estaría indicando un lapso pos-Tinguirirquense a pre-Deseadense, que estaría representando una nueva fauna predeseadense que completa parcialmente el hiatus entre la EM Tinguiririquense y la EM Deseadense (Carlini et al., 2010 y Ciancio, 2010).

Desde el punto de vista paleobiogeográfico Barrancas Blancas correspondería a la localidad más oriental de Patagonia con fauna paleógena de las Edades Deseadense, Tinguiririquense y del lapso pos-Tinguiririquense a pre-Deseadense. Finalmente aunque los taxones son típicamente patagónicos, adquiere reelevancia la presencia de afinidades con faunas deseadense de latitudes más bajas y taxones en común con la fauna de Tinguiririca en Chile central, lo que podría estar indicando cierta conexión paleobiogeográfica con faunas de edades equivalentes que se han desarrollado fuera de las áreas típicamente patagónicas.

\section{Agradecimientos}

Al Lic. M. Franchi (SEGEMAR) quien nos brindó los primeros datos del sitio paleontológico 'Barrancas Blancas'. A las familias Sardón y Siracusa, propietarias de la Estancia La Mugiana y la Estancia La Argentina (ex Lochiel), respectivamente, por permitirnos y facilitarnos los accesos al sitio de estudio. Al Dr. A. Monti (Universidad de la Patagonia S.J. Bosco) por su gran ayuda y colaboración en varias etapas de este trabajo y por su asesoramiento profesional en los aspectos geológicos. Al Sr. R. Taylor (CENPAT-CONICET) y al Sr. R. Vacca (Museo Paleontológico Egidio Feruglio), por su generoso y gran apoyo en las tareas de campo. A la Sra. L. Reiner y al Sr. E. Ruigómez (Museo Paleontológico Egidio Feruglio) por su apoyo en las tareas de preparación de los ejemplares fósiles y el acceso a las colecciones, respectivamente. A la Dra. E. Cerdeño (Instituto Argentino de Nivología, Glaciología y Ciencias Ambientales-Centro Regional de Investigaciones Científicas y Tecnológicas) por la lectura crítica del manuscrito. Al Dr. J. Gelfo, al Dr. M. Reguero (Museo La Plata) y a la Dra. A.M. Ribeiro (Universidad Federal do Río Grande do Soul) por el asesoramiento en varias determinaciones 
sistemáticas. A la Dra. G. Massaferro (CENPAT-CONICET) por la confección de los mapas y perfil geológico. Finalmente, a los árbitros, Lic. M. Bond y Dr. E. Bostelmann, quienes con sus aportes ayudaron a mejorar este trabajo. Este estudio se enmarca en el correspondiente Convenio con el Gobierno de la provincia del Chubut para trabajos de Colección e Investigación en yacimientos paleontológicos del Chubut y recibió el siguiente financiamiento: CONICET PIP 2628 y 2099 PICT-SECYT 07/32344.

\section{Referencias}

Ameghino, F. 1887. Enumeración sistemática de las especies de mamíferos fósiles coleccionados por Carlos Ameghino en los terrenos Eocenos de Patagonia austral y depositados en el Museo de La Plata. Boletín del Museo de La Plata 1: 1-26.

Ameghino, F. 1889. Contribución al conocimiento de los mamíferos fósiles de la República Argentina. Academia Nacional de ciencias de Córdiba. Actas 6: 1-1027.

Ameghino, F. 1891. Nuevos restos de mamíferos fósiles descubiertos por Carlos Ameghino en el Eoceno inferior de la Patagonia austral. Especies nuevas, adiciones y correcciones. Revista Argentina de Historia Natural 1: 289-328.

Ameghino, F. 1894a. Sur les oiseaux fossiles de Patagonie et la faune mammalogique des couches à Pyrotherium. Boletín del Instituto Geográfico Argentino 15: 501-660.

Ameghino, F. 1894b. Enumération synoptique des espèces mammifères fossiles des formations éocènes de Patagonie. Academia Nacional de Ciencias, Boletín 13: 259-452. Córdoba.

Ameghino, F. 1895. Première contribution à la connaissance de la faune mammalogique des couches à Pyrotherium. Boletín Instituto Geográfico Argentino 15: 603-660.

Ameghino, F. 1897. Les mamiferes crétacés de l'Argentine. Deuxieme contribution a la connaissance de la faune mammalogique des couches a Pyrotherium. Instituto Geográfico Argentino, Boletín 18: 405-521.

Ameghino, F. 1901. Notices préliminaires sur des ongulés noveaux des terrains Crètacès de Patagonie. Academia Nacional de Ciencias, Boletín 16: 349-426. Córdoba.

Ameghino, F. 1902. Notices préliminaires sur des mamiferes nouveaux des terrains Crètacès de Patagonie. Academia Nacional de Ciencias, Boletín 17: 5-70. Córdoba.

Ameghino, F. 1906. Les formations sédimentaires du crétacé supérieur et du tertiare de Patagonie avec un parallèle entre leurs faunaes mammalogiques et celles de L'ancien continent. Anales del Museo Nacional de Buenos Aires, Serie 3, 15: 1-658.
Andreis, R.R.; Mazzoni, M.M.; Spalletti, L.A. 1975. Estudio estratigráfico y paleoambiental de las sedimentitas terciarias entre Pico Salamanca y Bahía Bustamante, provincia de Chubut, República Argentina. Revista de la Asociación Geológica Argentina 30: 85-103.

Bellosi, E.S.; Laza, J.H.; Sánchez, M.V.; Genise, J.F. 2010. Ichnofacies analysis of the Sarmiento Formation (middle Eocene-early Miocene) at Gran Barranca, central Patagonia. In The paleontology of Gran Barranca: evolution and environmental change through the Middle Cenozoic of Patagonia (Madden, R.H.; Carlini, A.A.; Vucetich, M.G.; Kay, R.F.; editores). Cambridge University Press: 306-316. Cambridge.

Billet, G.; Patterson, B.; de Muizon, C. 2009. Craniodental anatomy of Late Oligocene archaeohyracids (Notoungulata, Mammalia) from Bolivia and Argentina and new phylogenetic hypotheses. Zoological Journal of the Linnean Society 155: 458-509.

Bond, M.; López, G. 1995. Los mamíferos de la Formación Casa Grande (Eoceno) de la provincia de Jujuy, Argentina. Ameghiniana 32 (3): 301-309.

Bond, M.; López, G.; Reguero, M. 1996. 'Astraponotéen plus supérieur' of Ameghino: another interval in the Paleogene record of South America. Journal of Vertebrate Paleontology, Supplement to 16 (3): 23A.

Bond, M.; López, G.; Reguero, M. 1997a. Rocas Bayas, una localidad fosilífera paleógena de la provincia de Río Negro, República Argentina. Ameghiniana 34 (4): 533.

Bond, M.; Reguero, M.; López, G.; Carlini, A.A.; Goin, F.; Madden, R.H.; Vucetich, M.G.; Kay, R.F. 1997b. The 'Astraponoteen plus superieur' (Paleogene) in Patagonia. Ameghiniana 34 (4): p. 533.

Bond, M.; López, G.; Reguero, M.A.; Scillato-Yané, G.J.; Vucetich, M.G. 1998. Los mamíferos de la Formación Fray Bentos (Edad-mamífero Deseadense, Oligoceno superior?) de las provincias de Corrientes y Entre Ríos, Argentina. Asociación Paleontológica Argentina, Publicación Especial 5, Paleógeno de América del Sur de la Península Antártica: 41-50.

Bordas, A.F. 1933. Notas sobre los Eutatinae: nueva subfamilia extinguida de Dasypodidae. Anales del Museo Nacional de Historia Natural de Buenos Aires 37: 583-614.

Bordas, A.F.1943. Contribución al conocimiento de las bentonitas argentinas. Apartado de la Revista Minera, Geología y Mineralogía, Tomo 14 (1-2): 1-60.

Bostelmann, E.; Ciancio, M.R.; Rinderknecht, A.; Perea, D. 2011. The Cingulata (Mammalia, Xenarthra) of the Fray Bentos Formation (Late Oligocene) of Uruguay. Ameghiniana 43 (4): R145-R146. 
Bown, T.M.; Fleagle, J.G. 1993. Systematics, Biostratigraphy and Dental Evolution of the Palaeothentidae, Later Oligocene to Early-Middle Miocene (DeseadanSantacrucian) Cenolestoid Marsupials of South America. Paleontological Society, Memoire 29. Journal of Paleontology, Supplement to 67 (2): 76 p.

Bown, T.; Fleagle, J.G.; Reed, K.E.; Dozo, M.T. 1993. The Eocene-Miocene Geology of Cabeza Blanca, a Vertebrate Fossil Locality in Southeast Chubut Province, Argentina. Journal of Vertebrate Paleontology, Supplement to 13 (3): p. 27A.

Camacho, H.H. 1979. Descripción geológica de la Hoja 47h-48g, bahía Camarones. Servicio Geológico Nacional de Argentina, Boletín 153: 27 p. Buenos Aires.

Carlini, A.A.; Scillato-Yané, G.J. 1999. Cingulata del Oligoceno tardío de Salla, Bolivia. In Congreso Internacional Evolución Neotropical del Cenozoico, Resúmenes: p. 15. La Paz.

Carlini, A.A.; Ciancio, M.; Scillato-Yané, G.J. 2004. La transición Eoceno-Oligoceno y su manifestación en la diversidad de los Cingulata (Mammalia, Xenarthra). Inferencias paleoecológicas. Ameghiniana, Suplemento Resúmenes 41 (4): 23-24R.

Carlini, A.A.; Ciancio, M.; Scillato-Yané, G.J. 2005. Los Xenarthra de Gran Barranca, más de 20 Ma de historia. In Congreso Geológico Argentino, No. 16, Actas 4: 419-424. La Plata.

Carlini, A.A.; Ciancio, M.R.; Zurita, A.E. 2007. Los Cingulata (Mammalia, Xenarthra, Dasypodidae) del Oligoceno de la provincia de Corrientes, Argentina y su importancia paleobiogeográfica. Ameghiniana 44 (4): 90-91R.

Carlini, A.A.; Ciancio, M.R.; Flynn, J.J.; Scillato-Yané, G.J.; Wyss, A.R. 2009. The phylogenetic and biostratigraphic significance of new armadillos (Mammalia, Xenarthra, Dasypodidae, Euphractinae) from the Tinguirirican (Early Oligocene) of Chile. Journal of Systematic Palaeontology 7 (4): 489-503.

Carlini, A.A.; Scillato-Yané, G.J.; Ciancio, M.R. 2010. Middle Eocene-early Miocene Dasypodidae (Xenarthra) of southern South America, successive faunas in Gran Barranca; biostratigraphy and palaeoecology. In The paleontology of Gran Barranca: evolution and environmental change through the Middle Cenozoic of Patagonia (Madden, R.H.; Carlini, A.A.; Vucetich, M.G.; Kay, R.F.; editores). Cambridge University Press: 106-129. Cambridge.

Castellanos, A. 1932. Nuevos géneros de gliptodontes en relación con su filogenia. Physis 11 (38): 92-100.

Cerdeño, E. 2011. Quebrada Fiera (Mendoza), an impor- tant paleobiogeographic center in the South American late Oligocene. Estudios geológicos 67 (2): 375-384.

Cerdeño, E.; Vera, B. 2010. Mendozahippus fierensis, gen. et sp. nov., new Notohippidae (Notoungulata) from the late Oligocene of Mendoza (Argentina). Journal of Vertebrate Paleontology 30 (6): 1805-1817

Chaffee, R.G. 1952. The Deseadan vertebrate fauna of the Scarritt Pocket, Patagonia. Bulletin of the American Museum of Natural History 98: 507-562.

Ciancio, M.R. 2010. Los Dasypodoidea (Mammalia, Xenarthra) del Deseadense (Oligoceno) de América del Sur. Su importancia filogenética y bioestratigráfica. Tesis Doctoral (Inédita), Facultad de Ciencias Naturales y Museo, Universidad Nacional de La Plata, No. 1098: 290 p.

Ciancio, M.R.; Carlini, A.A. 2007. Morfología del sistema pilífero en Eutatinos (Xenarthra, Dasypodidae) del Oligoceno de Patagonia (Argentina). In Congreso de Mastozoología en Bolivia, No. 3, Resúmenes: 49-50. Santa Cruz de La Sierra.

Ciancio, M.R.; Carlini, A.A. 2008. Identificación de Ejemplares Tipo de Dasypodidae (Mammalia, Xenarthra) del Paleógeno de Argentina. Revista del Museo Argentino de Ciencias Naturales 10 (2): 221-237.

Cifelli, R.L. 1985. Biostratigraphy of the Casamayoran, Early Eocene, of Patagonia. American Museum Novitates 2820: 1-26.

Cope, E. 1889. The Edentata of North America. American Naturalist 23: 657-664.

Croft, D.A.; Bond, M.; Flynn, J.J.; Reguero, M.A.; Wyss, A.R. 2003. Large archaeohyracids (Typotheria, Notoungulata) from central Chile and Patagonia including a revision of Archaeotypotherium. Fieldiana 49: 1-38.

Croft, D.A.; Flynn, J.J.; Wyss, A.R. 2007. A new basal glyptodontid and other Xenarthra of the early Miocene Chucal Fauna, northern Chile. Journal of Vertebrate Paleontology 27: 781-797.

Croft, D.; Flynn, J.J.; Wyss, A.R. 2008. The Tinguiririca fauna of Chile and the early stages of 'modernization' of South American Mammal faunas. Arquivos do Museu Nacional 66: 191-211.

Dingle, R.V.; Lavelle, M. 1998. Late Cretaceous-Cenozoic clímatic variations of the northern Antartic Península; new geochemical evidence and review. Palaeogeography, Palaeoclimatology, Palaeoecology 141: 215-232.

Dozo, M.T.; Vera, B. 2010. First skull and associated postcranial bones of Macraucheniidae (Mammalia, Litopterna) from the Deseadan SALMA (late Oligocene) of Cabeza Blanca (Chubut, Argentina). Journal of Vertebrate Paleontology 30 (6): 1818-1826. 
Dozo, M.T.; Monti, A.; Marani, H.; Vacca, R.; ScillatoYané, G.J. 2004. Barrancas Blancas, una nueva localidad fosilífera del Paleógeno del Chubut, Argentina. Ameghiniana, Suplemento Resúmenes 41 (4): p. 45R.

Engelmann, G.F. 1987. A new Deseadan sloth (Mammalia: Xenarthra) from Salla, Bolivia, and its implications for the primitive condition of the dentition in edentates. Journal of Vertebrate Paleontology 7 (2): 217-223

Feruglio, E. 1949. Descripción Geológica de la Patagonia. Buenos Aires: Dirección General de Yacimientos Petrolíferos Fiscales II: 1-349.

Feruglio, E. 1950. Descripción Geológica de la Patagonia. Buenos Aires: Dirección General de Yacimientos Petrolíferos Fiscales III: 1-431.

Flores, M.A. 1956. Reconocimiento geológico entre Malaspina y Camarones. Chubut. Yacimientos Petrolíferos Fiscales 200: 15 p.

Flynn, J.J.; Swisher III, C.C. 1995. Cenozoic South American land mammal ages: correlation to global geochronologies. In Geochronology, Time Scales, and Global Stratigraphic Correlation (Berggren, W.A.; Kent, D.V.; Aubry, M.P.; Hardenbol, J.; editores), Society for Sedimentary Geology: 317-333. Tulsa.

Flynn, J.J.; Wyss, A.R.; Croft, D.A.; Charrier, R. 2003. The Tinguiririca Fauna, Chile: biochronology, palaeoecology, biogeography, and a new earliest Oligocene South American Land Mammal 'Age'. Palaeogeography, Palaeoclimatology, Palaeoecology 195: 229-259.

Gaudry, A. 1906. Fossiles de Patagonie. Les attitudes de quelques animaux. Annales de Paléontologie 1: 1-42.

Goin, F.J.; Abello, M.A.; Chornogubsky, L. 2010. Middle Tertiary marsupials from central Patagonia (early Oligocene of Gran Barranca): understanding South's America Grande Coupure. In The paleontology of Gran Barranca: evolution and environmental change through the Middle Cenozoic of Patagonia (Madden, R.H.; Carlini, A.A.; Vucetich, M.G.; Kay, R.F.; editores). Cambridge University Press: 69-92. Cambridge.

Gray, J.E. 1821. On the natural arrangement of vertebrose animal. London Medical Repository 5: 296-310.

Gray, J.E. 1869. Catalogue of Carnivorous, Pachydermatous, and Edentata. Mammalia in the British Museum. British Museum: 398 p. London.

Hitz, R.; Reguero, M.A.; Wyss, A.R.; Flynn, J.J. 2000. New Interatheriines (Interatheriidae, Notoungulata) from the Paleogene of central Chile and southern Argentina. Fieldiana (Geology) New Series 42: 1-26.

Illiger, J.K.W. 1811. Prodromus systemalis mammalium et avium additis terminis zoographicis utriusque classis: 301 p. Berlin.
Kay, R.F.; MacFadden, B.J.; Madden, R.H.; Sandeman, H.; Anaya, F. 1998. Revised age of the Salla beds, Bolivia, and its bearing on the age of the Deseadan South American Land Mammal Age. Journal of Vertebate Paleontology 18 (1): 189-199.

Kay, R.F.; Madden, R.H.; Vucetich, M.G.; Carlini, A.A.; Mazzoni, M.M.; Ré, G.H.; Heizler, M.; Sandeman, H. 1999. Revised geochronology of the Casamayoran South American Land Mammal Age: Climatic and biotic implications. Proceeding of the National Academy of Science of the United States of America 96: 13235-13240.

Kramarz, A.G.; Vucetich, M.G.; Carlini, A.A.; Ciancio, M.R.; Abello, M.A.; Deschamps, C.M.; Gelfo, J. 2010. A new mammal fauna at the top of the sequence at Gran Barranca: its biochronological meaning. In The paleontology of Gran Barranca: evolution and environmental change through the Middle Cenozoic of Patagonia (Madden, R.H.; Carlini, A.A.; Vucetich, M.G.; Kay, R.F.; editores). Cambridge University Press: 264-276. Cambridge.

Lema, H.; Busteros, A.; Franchi, M. 2001. Hoja Geológica 4466 II y IV Camarones, Provincia del Chubut. Servicio Geológico Minero Argentino, Instituto de Geología y Recursos Minerales, Boletín 261: 60 p.

Linnaeus, C.V. 1758. Sistema Naturae per Regna Tria Naturae. 10 th edition, 1, Regnum Animale. L. Salvii, Ho, miae, Sweden.

Loomis, F. 1914. The Deseado Formation of Patagonia. The Rumford Press: 232 p. Concord.

López, G.M.; Ribeiro, A.M.; Bond, M. 2010. The Notohippidae (Mammalia, Notoungulata) from Gran Barranca: preliminary considerations. In The paleontology of Gran Barranca: evolution and environmental change through the Middle Cenozoic of Patagonia (Madden, R.H.; Carlini, A.A.; Vucetich, M.G.; Kay, R.F.; editores). Cambridge University Press: 143-151. Cambridge.

Madden, R. 1990. Miocene Toxodontidae (Notoungulata, Mammalia) from Colombia, Ecuador, and Chile. Ph.D. Thesis (Inédito), Duke University: 407 p. Durham.

Madden, R.H.; Carlini, A.A.; Vucetich, M.G.; Kay, R.F. (editores). 2010. The paleontology of Gran Barranca: evolution and environmental change through the Middle Cenozoic of Patagonia. Cambridge University Press: 448 p. Cambridge.

Marani, H.A. 2005. Los Rhynchippinae (Mammalia, Notoungulata: Toxodontia: Notohippidae) de la edad Deseadense de Cabeza Blanca (Chubut, Argentina): revisión sistemática y filogenia. Tesis de Licenciatura 
(Inédito), Facultad de Ciencias Naturales, Universidad Nacional San Juan Bosco: 72 p. Puerto Madryn.

Marani, H.; Dozo, M.T. 2008. El cráneo más completo de Eurygenium latirostris Ameghino, 1895 (Mammalia, Notoungulata), un Notohippidae del Deseadense (Oligoceno tardío) de la Patagonia, Argentina. Ameghiniana 45 (3): 619-626.

Marshall, L.; Pascual, R. 1977. Nuevos marsupiales Caenolestidae del 'Piso Notohippidense' (SO de Santa Cruz, Patagonia) de Ameghino. Sus aportaciones a la cronología y evolución de las comunidades de mamíferos sudamericanos. Publicaciones del Museo Municipal de Ciencias Naturales de Mar del Plata 'Lorenzo Scaglia' 2 (4): 91-122.

Marshall, L.; Pascual, R. 1978. Una escala temporal radiométrica preliminary de las Edades-mamífero del Cenozoico medio y tardío sudamericano. Obras del Centenario del Museo de La Plata 5: 11-28.

Marshall, L.; Sempere, T. 1991. The Eocene to Pleistocene vertebrates of Bolivia and their stratigraphic context: a review. In Fósiles y Facies de Bolivia-Vol. 1, Vertebrados (Suárez, R.; editor). Revista Técnica de YPFB (Yacimientos Petrolíferos Fiscales Bolivianos) 12 (3-4): 631-652.

Marshall, L.G.; Hoffstetter, R.; Pascual, R. 1983. Mammals and stratigraphy: geochronology of the continental mammal-bearing Tertiary of South America. Palaeovertebrata, Mémoire Extraordinaire 1983: 1-93.

Marshall, L.G.; Cifelli, R.L.; Drake, R.E.; Curtis, G.H. 1986. Vertebrate Paleontology, Geology and Geocronology of the Tapera de López and Scarrit Pocket, Chubut province, Argentina. Journal of Paleontology 60 (4): 920-951.

McKenna, M.C.; Wyss, A.R.; Flynn, J.J. 2006. Paleogene pseudoglyptodont xenarthrans from central Chile and Argentine Patagonia. American Museum Novitates 3536: 1-18.

Owen, R. 1842. Ueber das Mylodon und das Glyptodon, zwei in Südamerika neu abgefundene fossile Vierfüsser. Neue Notizen aus dem Gebiete der Natur und Heilkunde 23 (2, 486): 17-21.

Owen, R. 1853. Description of some species of the extinct genus Nesodon, with remarks on the primary group (Toxodontia) of the hoffed quadrupeds to which that genus is referable. Philosophical Transactions of the Royal Society of London 143: 291-309.

Pascual, R. 1984. La sucesión de las edades mamífero, de los climas y del diastrofismo sudamericano durante el Cenozoico: fenómenos concurrentes. Anales de la Academia Nacional Ciencias Exactas, Físicas y Naturales 36: 15-37.
Pascual, R.; Odreman Rivas, O. 1973. Las unidades estratigráficas del Terciario portadoras de mamíferos. Su distribución y sus relaciones con los acontecimientos diastróficos. In Congreso Geológico Argentino, No. 5 , Actas 3: 293-338. Buenos Aires.

Pascual, R.; Hinojosa, E.; Gondar, D.; Tonni, E. 1965. Las edades del Cenozoico mamalífero de la Argentina, con especial atención a aquellas del territorio bonaerense. Anales de la Comisión de Investigaciones Científicas de la Provincia de Buenos Aires, La Plata 6: 165-193.

Pascual, R.; Vucetich, M.G.; Scillato-Yané, G.J.; Bond, M. 1985. Main pathways of mammal diversification in South America. In The Great American Biotic Interchange (Stehli, F.G.; Webb, S.D.; editores). Plenum Press: 219-247. New York.

Pascual, R.; Ortiz Jaureguizar, E.; Prado. J.L. 1996. Land Mammals: Paradigm for Cenozoic South American Geobiotic Evolution. In Contributions of Southern South America to Vertebrate Paleontology (Arratia, G.; editor). Münchner Geowissenschaftliche Abhandlungen (A) 30: 265-319.

Pascual, R.; Carlini, A.A.; Bond, M.: Goin, F.J. 2002. Mamíferos cenozoicos. Geología y Recursos Naturales de Santa Cruz (Haller, M.J.; editor). In Congreso Geológico Argentino, No. 15, Relatorio: 533-544. El Calafate.

Patterson, B. 1934. Upper premolar-molar structure in the Notoungulata with notes on Taxonomy. Field Museum of Natural History 6: 91-111.

Prothero, D.R. 1994. The Eocene-Oligocene Transition: Paradise Lost. Columbia University Press: 291 p.

Pujos, F.; De Iuliis, G. 2007. Late Oligocene Megatherioidea Fauna (Edentata: Xenarthra) from Salla-Luribay (Bolivia): new data on basal sloth radiation and Cingulata-Phyllophaga split. Journal of Vertebrate Paleontology 27 (1): 132-144.

Ré, G.; Bellosi, E.S.; Heizler, M.; Vilas, J.F.; Madden, R.H.; Carlini, A.A.; Kay, R.F.; Vucetich, M.G. 2010. A geochronology for the Sarmiento Formation at Gran Barranca. In The paleontology of Gran Barranca: evolution and environmental change through the Middle Cenozoic of Patagonia (Madden, R.H.; Carlini, A.A.; Vucetich, M.G.; Kay, R.F.; editores). Cambridge University Press: 46-58. Cambridge.

Reguero, M.A. 1993. Los Typotheria y los Hegetotheria (Mammalia, Notoungulata) eocenos de la localidad Cañadón Blanco, Chubut. Ameghiniana 30 (3): 336.

Reguero, M.A. 1999. El problema de las relaciones sistemáticas y filogenéticas de los Typotheria y He- 
getotheria (Mammalia, Notoungulata): análisis de los taxones de Patagonia de la Edad-mamífero Deseadense (Oligoceno). Tesis Doctoral (Inédita), Universidad de Buenos Aires, 2 tomos. Buenos Aires.

Reguero, M.A.; Escribano, V. 1996. Trachytherus spegazzinianus Ameghino, 1889 (Notoungulata, Mesotheridae) de la edad Deseadense (Oligoceno superior-Mioceno inferior) de Argentina y Bolivia. Naturalia Patagónica, Ciencias de la Tierra 4: 43-71.

Reguero, M.A.; Prevosti, F. 2010. Rodent-like notoungulates (Typotheria) from Gran Barranca, Chubut Province, Argentina: phylogeny and systematics. In The paleontology of Gran Barranca: evolution and environmental change through the Middle Cenozoic of Patagonia (Madden, R.H.; Carlini, A.A.; Vucetich, M.G.; Kay, R.F.; editores). Cambridge University Press: 152-169. Cambridge.

Reguero, M.A.; Ubilla, M.; Perea, D. 2003. A new species of Eopachyrucos (Mammalia, Notoungulata, Interatheriidae) from the Late Oligocene of Uruguay. Journal of Vertebrate Paleontology 23 (2): 445-457.

Reguero, M.; Dozo, M.T.; Cerdeño, E. 2007. A poorly known rodentlike mammal (Pachyrukhinae, Hegetotheriidae, Notoungulata) from the Deseadan (Late Oligocene) of Argentina. Paleoecology, Biogeography and radiation of the rodentlike ungulates in South America. Journal of Paleontology 81 (6): 1301-1307.

Reguero, M.A.; Croft, D.C.; López, G.M.; Alonso, R.N. 2008. Eocene archaeohyracids (Mammalia: Notoungulata: Hegetotheria) from the Puna, northwest Argentina. Journal of South American Earth Sciences 26: 225-233.

Ribeiro, A.M.; López, G.; Bond, M. 2010. The Leontiniidae (Mammalia, Notoungulata) from the Sarmiento Formation at Gran Barranca, Chubut Province, Argentina. In The paleontology of Gran Barranca: evolution and environmental change through the Middle Cenozoic of Patagonia (Madden, R.H.; Carlini, A.A.; Vucetich, M.G.; Kay, R.F.; editores). Cambridge University Press: 170-181. Cambridge.

Roth, S. 1901. Notas sobre algunos nuevos mamíferos fósiles. Revista del Museo de La Plata 10: 252-256.

Roth, S. 1903. Los ungulados sudamericanos. Anales del Museo La Plata (Sección Paleontología) 5: 1-36.

Salamy, K.A.; Zachos, J.C. 1999. Latest Eocene-Early Oligocene climate change and Southern Ocean fertility: inferences from sediment accumulation and stable isotope data. Palaeogeography, Palaeoclimatology and Palaeoecololgy 145: 1-77.

Scillato-Yané, G. J. 1986. Los Xenarthra fósiles de la Argentina (Mammalia, Edentata). In Congreso Argentino de Paleontología y Bioestratigrafía, No 4, Actas 2: 151-155. Mendoza.

Sciutto, J.C.; Césari, O.; Escribano, V.; Pezzuchi, H. 2000. Hoja Geológica 4566 II Comodoro Rivadavia, Provincia del Chubut. Servicio Geológico Minero Argentino, Instituto de Geología y Recursos Minerales, Boletín 244: 53 p.

Shockey, B.J. 1997. Two new notoungulates (Family Notohippidae) from the Salla Beds of Bolivia (Deseadan: Late Oligocene): systematics and functional morphology. Journal of Vertebrate Paleontology 17 (3): 584-599.

Shockey, B.J.; Salas Gismondi, R.; Gans, P.; Jeong, A.; Flynn, J.J. 2009. Paleontology and Geochronology of the Deseadan (late Oligocene) of Moquegua, Perú. American Museum Novitates 3668: 1-24.

Shockey, B.J.; Flynn, J.J.; Croft, D.A.; Gans, P.; Wyss, A.R. 2012. New leontiniid Notoungulata (Mammalia) from Chile and Argentina: comparative anatomy, character analysis, and phylogenetic hypotheses. American Museum Novitates 3737: 1-64.

Simpson, G. 1941. The Eogene of Patagonia. American Museum Novitates 1120:1-15.

Simpson, G.G. 1945. The principles of classification and a classification of mammals. Bulletin American Museum of Natural History 85: 1-350.

Simpson, G.G. 1948. The beginning of the age of mammals in South America. Part 1. Bulletin of theAmerican Museum of Natural History 91 (1): 1-232.

Simpson, G.G. 1967. The beginning of the Age of the Mammals in South America. Part II. Bulletin of the American Museum of Natural History 137: 1-259.

Soria, M.F. 2001. Los Proterotheriidae (Litopterna, Mammalia), sistemática, origen y filogenia. Monografías del Museo Argentino de Ciencias Naturales 1: 1-167.

Spalletti, L.; Mazzoni, M. 1977. Sedimentología del Grupo Sarmiento en el Perfil Ubicado al Sudeste del Lago Colhue Huapi, Provincia de Chubut. Obra del Centenario del Museo de La Plata, Tomo IV: 261-283

Stehlin, H.G. 1909. Remarques sur les faunules de mammifères des couches eocènes et oligocènes du Bassin de Paris. Bulletin de la Société Géologique de France, série 4 (9): 488-520.

Tournoüer, A.1903. Note sur la géologie et la paléontologie de la Patagonie. Bulletin de la Société Géologique de France, série 4 (3): 463-473.

Ubilla, M.; Perea, D.; Bond, M. 1994. The deseadan land mammal age in Uruguay and the report of Scarrittia robusta nov. sp. (Leontiniidae, Notoungulata) in the Fray Bentos Formation (Oligocene-?Lower miocene). Geobios 27 (1): 95-102. 
Ubilla, M.; Perea, D.; Bond, M. 1999. Two new records of Notoungulates (Isotemnidae; Oldfieldthomasiidae n.g., n. sp.) from Fray Bentos Fm. (Deseadan SALMA, Oligocene) in the Santa Lucía Basin, Uruguay. In Congreso Internacional Evolución Neotropical del Cenozoico, Resúmenes: p. 43. La Paz.

Vucetich, M.G.; Ribeiro, A.M. 2003. A new and Primitive Rodent from The Tremembé Formation (Late Oligocene) of Brazil, with Comments on the Morphology of the Lower Premolars of Caviomorph Rodents. Revista Brasileira de Paleontologia 5: 73-82.

Vucetich, M.G.; Vieytes, E.C.; Pérez, M.E.; Carlini, A.A. 2010. The rodents from La Cantera and the early evolution of caviomorphs in South America. In The paleontology of Gran Barranca: evolution and environmental change through the Middle Cenozoic of Patagonia (Madden, R.H.; Carlini, A.A.; Vucetich, M.G.; Kay, R.F.; editores). Cambridge University Press: 93-205. Cambridge.
Windhausen, A. 1931. Geología Argentina. In Geología Histórica y Regional del Territorio Argentino (Segunda Parte). Editorial Peuser: 1-40. Buenos Aires.

Winge, H. 1923. Pattedyr-Slaegter. 1-Monotremata, Marsupialia, Insectivora, Chiroptera, Edentata. Copenhagen, Hagarup: 360 p.

Wolfe, J.A. 1978. A paleobotanical interpretation of Tertiary climates in the northern Hemisphere. American Scientist 66: 694-703.

Wood, A.E.; Patterson, B. 1959. The rodents of the Deseadan Oligocene of Patagonia and the beginnings of South American rodent evolution. Bulletin Museum of Comparative Zoology 120: 281-428.

Yllañez, E. 1979. Descripción Geológica de la Hoja 46f Uzcudum, Provincia del Chubut. Dirección Nacional de Servicio Geológico: 58 p. Buenos Aires.

Zachos, J.; Pagani, M.; Sloan, L.; Thomas, E.; Billups, K. 2001. Trends, rhythms, and aberrations in global climate $65 \mathrm{Ma}$ to present. Science 292: 686-693.

Manuscript received: August 09, 2012; revised/accepted: July 05, 2013; available online: July 08, 2013. 